\title{
Les classes d'Eisenstein des variétés de Hilbert-Blumenthal
}

\author{
David Blottière \\ Universität Paderborn, \\ Institut für Mathematik, \\ Warburger Str. 100, \\ 33098 Paderborn, Germany. \\ email : blottier@math.upb.de
}

\begin{abstract}
Résumé
Dans [B2], on a démontré que les courants de Levin (cf. [L]) permettent de décrire explicitement les classes d'Eisenstein d'un schéma abélien au niveau topologique. On applique ici ce résultat, conjecturé par Levin, au cas où le schéma abélien est une famille de variétés abéliennes de Hilbert-Blumenthal (cf. Proposition4.3). On étudie ensuite la dégénérescence de ces classes d'Eisenstein en une pointe de la compactification de Baily-Borel de la variété de Hilbert-Blumenthal. Au moyen du Théorème de Burgos-Wildeshaus [BW, Theorem 2.9], on démontre un résultat de rigidité (cf. Proposition 3.4) qui permet de restreindre l'étude au niveau topologique. On prouve, en utilisant la description explicite des classes d'Eisenstein obtenue précédemment, que ces classes dégénèrent en des valeurs spéciales d'une fonction $L$ associée au corps de nombres totalement réel sous-jacent (Théorème 5.2). On en déduit une preuve géométrique du Théorème de Klingen-Siegel (Corollaire 5.3) et un résultat de non annulation pour certaines de ces classes d'Eisenstein (Corollaire 5.4).
\end{abstract}

\begin{abstract}
In [B2], we have proved that Levin's currents (cf. [L]) give an explicit description of the Eisenstein classes of an abelian scheme at the topological level. We apply here this result, conjectured by Levin, in the situation where the abelian scheme is an Hilbert-Blumenthal family of abelian varieties (cf. Proposition 4.3). Then we study the degeneration of these Eisenstein classes at a cusp of the Baily-Borel compactification of the Hilbert-Blumenthal variety. Using the Theorem of Burgos-Wildeshaus [BW, Theorem 2.9], we prove a rigidity result which allows us to restrict the study at the topological level (cf. Proposition 3.4). We show, using the explicit description of the Eisenstein classes obtained previously, that these classes degenerate in special values of an $L$-function associated to the underlying totally real number field (Théorème 5.2). We deduce then a geometric proof the KlingenSiegel Theorem (Corollaire 5.3) and a non vanishing result for some of these Eisenstein classes (Corollaire 5.4).
\end{abstract}




\section{Introduction}

Les classes d'Eisenstein d'un schéma abélien (cf. [B2, Partie 5] pour une définition) ont une origine motivique d'après Kings (cf. [Ki1]). Dans le cas elliptique, celles-ci ont été intensivement étudiées et on en connaît des propriétés remarquables, mais en dimension supérieure, peu de choses sont connues à leur sujet. Par exemple, la non nullité de ces classes constitue un problème ouvert. Levin avait conjecturé que les courants qu'il construit dans $\mathrm{L}$ permettent de décrire le polylogarithme (cf. [B2, Partie 4.1] pour une définition) et par suite les classes d'Eisenstein d'un schéma abélien. Cette conjecture étant démontrée (cf. [B2]), on peut envisager d'utiliser cet outil pour aborder l'étude des classes d'Eisenstein en dimension supérieure.

Dans cet article, nous considérons le cas particulier des familles de variétés abéliennes de Hilbert-Blumenthal et nous utilisons le-dit outil pour exhiber des propriétés remarquables des classes d'Eisenstein. Le lecteur pourra consulter la note [B1] pour un exposé concis des travaux présentés ici. Toutefois, dans la Proposition 3.1 et dans le Théorème 4.1 a) de [B1], les constantes rationnelles sont erronées. Les valeurs correctes de celles-ci sont données respectivement dans la Proposition 4.3 et le Théorème 5.2. On commence par donner une expression en coordonnées de ces classes, au niveau topologique (cf. Proposition 4.3). Celles-ci vivent dans un groupe de cohomologie de la base du schéma abélien, base qui est une variété de Hilbert-Blumenthal. On étudie ensuite leur dégénérescence en une pointe de la compactification de Baily-Borel de la base et l'on démontre que les résidus en cette pointe de ces classes s'expriment en termes de valeurs spéciales d'une certaine fonction $L$ attachée au corps de nombres totalement réel sous-jacent (cf. Théorème 5.2). On réduit ce calcul à un calcul au niveau topologique (cf. Proposition 3.4) en utilisant de façon essentielle le Théorème de Burgos-Wildeshaus [BW, Theorem 2.9]. Ce résidu étant un nombre rationnel, on en déduit une preuve géométrique du Théorème de Klingen-Siegel (cf. Corollaire 5.3).

Plusieurs preuves de ce Théorème ont été données depuis 1962, date de la publication de la première démonstration dans l'article [Kl]. Si notre approche présente une certaine analogie avec la preuve originale, elle met toutefois en lumière un lien géométrique nouveau avec la famille modulaire de Hilbert-Blumenthal, via le polylogarithme. On remarque que les deux démonstrations les plus récentes de ce résultat dues à Nori $[\mathrm{No}]$ et Sczech $[\mathrm{Sc}]$ utilisent toutes deux la rationalité d'une classe de cohomologie pour en déduire la rationalité d'une valeur spéciale de la fonction $L$ en question. Notre approche présente donc également une certaine analogie avec les leurs.

On déduit également de la forme particulière de la dégérescence des classes d'Eisenstein un résultat de non nullité pour certaines classes d'Eisenstein dans cette situation géométrique (cf. Corollaire (5.4). La preuve utilise une équation fonctionnelle due à Siegel pour la fonction $L$ qui apparaît dans l'expression du résidu. On mentionne enfin que Kings dans la prépublication Ki2 étudie également la dégénérescence des classes d'Eisenstein dans le cas Hilbert-Bimenthal.

On passe maintenant au contenu des différentes parties de cet article.

- Partie 2 : On définit le schéma abélien que l'on considère à l'aide du formalisme de Pink (cf. $[\mathrm{P}]$ ). Cette approche nous est utile pour la suite. On définit également des sections de torsion et on esquisse la construction de la compactification de Baily-Borel d'une composante connexe de la base. 
- Partie 3 : Cette partie est consacrée à la définition et à l'étude du morphisme résidu en une pointe de la compactification de Baily-Borel de la base du schéma abélien précédemment défini. On y montre en particulier que le but de ce morphisme est soit nul, soit canoniquement isomorphe à $\mathbb{Q}$. C'est ici qu'intervient le Théorème de Burgos-Wildeshaus BW, Theorem 2.9]. Son énoncé faisant appel au formalisme de Pink, la définition du schéma abélien dans ce langage nous est précieuse. On établit également un résultat de rigidité (cf. Proposition 3.4) qui nous permet de réduire le calcul du résidu à un calcul au niveau topologique.

- Partie 4 : Il s'agit ici de calculer les différents objets qui interviennent dans la définition des courants de Levin (cf. [L ). Le lecteur trouvera une explication des différents objets en jeu en début de section. Ces calculs effectués, on peut alors donner une formule pour les classes d'Eisenstein, au niveau topologique, dans notre contexte (cf. Proposition 4.3).

- Partie 5 : Disposant d'une expression explicite au niveau topologique des classes d'Eisenstein et d'un résultat de rigidité qui nous permet d'effectuer le calcul au niveau topologique, on détermine, dans cette partie, le résidu de ces classes en intégrant celles-ci le long du bord d'un voisinage ouvert de la pointe considérée. On établit alors le lien entre ces résidus et des valeurs spéciales d'une certaine fonction $L$ (cf. Notation 5.1 et Théorème 5.2) et on en déduit le Théorème de Klingen-Siegel (cf. Corollaire 5.3) et un résultat de non annulation (cf. Corollaire 5.4).

\section{Remerciements}

La dégénérescence des classes d'Eisenstein des familles modulaires de Hilbert-Blumenthal en des valeurs spéciales de fonctions $L$ du corps du nombres totalement réel sous jacent avait été conjecturée par Jörg Wildeshaus. Je tiens à lui exprimer toute ma gratitude pour m'avoir fait part de cette intuition, ainsi que pour les entretiens qu'il a bien voulu m'accorder. Je suis également ravi de remercier Guido Kings pour les discussions que nous avons partagées sur les résultats de cet article, lors de mon séjour à l'Université de Regensburg. Enfin, j'adresse un grand merci à Daniel Barsky qui a eu la gentillesse de bien vouloir répondre à mes questions sur les fonctions $L$ considérées dans ce travail.

\section{Notations :}

Pour $X$ un schéma de type fini, séparé et lisse sur $\mathbb{C}$, on note :

$\operatorname{VSHM}(X)$ la catégorie des $\mathbb{Q}$-variations de structures de Hodge mixtes polarisables et admissibles (cf. [Ka]) sur $X$,

$\overline{\mathbb{V}} \quad$ le système local sous-jacent à $\mathbb{V}$ pour $\mathbb{V} \in O b(\operatorname{VSHM}(X))$,

$\operatorname{MHM}(X) \quad$ la catégorie des $\mathbb{Q}$-modules de Hodge algébriques mixtes sur $X$ (cf. [Sa]).

En particulier, $M H M(\operatorname{Spec}(\mathbb{C}))$ est la catégorie des $\mathbb{Q}$-structures de Hodge mixtes polarisables, catégorie que l'on note simplement $S H M$.

On a un foncteur canonique $\operatorname{VSHM}(X) \rightarrow M H M(X)$ qui est exact, pleinement fidèle et grâce auquel on identifie $\operatorname{VSHM}(X)$ à une sous-catégorie pleine de $M H M(X)$.

Par construction de $M H M(X)$, on dispose d'un foncteur For := real $\circ$ rat: $D^{b} M H M(X) \rightarrow$ 
$D_{c}^{b}(X)$, où rat est le foncteur défini par M. Saito, real est le foncteur de Beilinson et $D_{c}^{b}(X)$ la sous catégorie pleine de la catégorie dérivée bornée des faisceaux de $\mathbb{Q}$-vectoriels sur $X(\mathbb{C})$, équipé de la topologie transcendante, dont les objets sont les complexes à cohomologie algébriquement constructible.

Convention 1.1 - Si $\mathbb{V} \in O b(\operatorname{VSH} M(X))$, For $(\mathbb{V})$ est le système local $\overline{\mathbb{V}}$ convenablement décalé. Dans cet article, on ne tient pas compte du décalage.

On fixe pour la suite :

$L \quad$ un corps de nombres totalement réel $L$ de dimension $g$,

$\left(\sigma_{k}\right)_{1 \leq k \leq g}$ une énumération des plongements réels de $L$,

$\mathfrak{a} \quad$ un idéal entier de $L$,

$N \quad$ un nombre entier plus grand que 3.

Soient $\mathfrak{b}$ un idéal fractionnaire de $L$ et $\mathfrak{p}$ un idéal premier de $L$. On note

$\mathcal{O}_{L} \quad$ l'anneau des entiers de $L$,

$\operatorname{Tr}_{L} \quad$ la trace de $L$,

$\mathrm{N}_{L} \quad$ la norme de $L$,

$d_{L} \quad$ le discriminant de $L$,

$\mathfrak{d}_{L} \quad$ la différente de $L$,

$\mathcal{N}_{L}(\mathfrak{b})$ la norme de $\mathfrak{b}$,

$\mathfrak{b}^{\vee} \quad$ le dual de $\mathfrak{b}$ relativement à $\operatorname{Tr}_{L}$,

$L_{\mathfrak{p}} \quad$ la complétion de $L$ en la valuation associée à $\mathfrak{p}$,

$\mathcal{O}_{L_{\mathfrak{p}}} \quad$ l'anneau des entiers de $L_{\mathfrak{p}}$,

$\mathfrak{b}_{\mathfrak{p}} \quad$ la complétion de $\mathfrak{b}$ en la valuation associée à $\mathfrak{p}$.

\section{Données géométriques}

On commence par définir le schéma abélien que l'on considère dans cet article. On utilise pour cela le formalisme et les résultats de la Thèse de Pink $[\mathrm{P}$. On rappelle ensuite rapidement la construction de la compactification de Baily-Borel de la base du schéma abélien précédemment défini, qui est une variété de Hilbert-Blumenthal. On définit enfin des sections de torsion.

Convention 2.1 - Soit $H$ un groupe algébrique sur $L$ et soit $K \in\{\mathbb{R}, \mathbb{C}\}$. Pour toute $K$-algèbre $A$, on dispose d'un isomorphisme de $K$-algèbres $A \otimes_{\mathbb{Q}} L \stackrel{\sim}{\rightarrow} A^{g}, a \otimes l \mapsto\left(\sigma_{k}(l) a\right)_{1 \leq k \leq g}$ au moyen duquel on identifie $\left(\operatorname{Res}_{L / \mathbb{Q}} H\right) \times_{\mathbb{Q}} K$ et $\left(H \times_{\mathbb{Q}} K\right)^{g}$.

\subsection{Définition de la base du schéma abélien}

Soit $i: \mathbb{G}_{m, \mathbb{Q}} \hookrightarrow \operatorname{Res}_{L / \mathbb{Q}} \mathbb{G}_{m, L}$ le morphisme qui à une $\mathbb{Q}$-algèbre $A$ fait correspondre le morphisme de groupes $A^{\times} \rightarrow(A \otimes L)^{\times}, a \mapsto a \otimes 1$. On définit le $\mathbb{Q}$-schéma en groupes réductif $G$ par le diagramme cartésien suivant : 


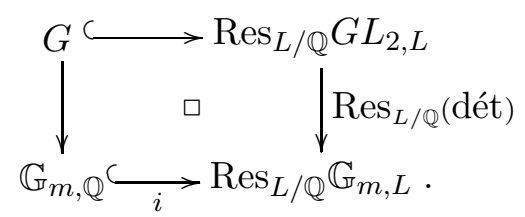

On pose $\mathfrak{H}^{ \pm}:=\mathbb{C}-\mathbb{R}$ et on fait de $\left(\mathfrak{H}^{ \pm}\right)^{g}$ un $G(\mathbb{R})$-espace homogène à gauche en faisant agir le groupe $G(\mathbb{R}) \subseteq G L_{2}(\mathbb{R})^{g}$ à gauche sur $\left(\mathfrak{H}^{ \pm}\right)^{g}$ via les homographies. On définit alors un morphisme $h:\left(\mathfrak{H}^{ \pm}\right)^{g} \rightarrow \operatorname{Hom}\left(\mathbb{S}_{\mathbb{C}}, G_{\mathbb{C}}\right) \subseteq \operatorname{Hom}\left(\mathbb{S}_{\mathbb{C}},\left(G L_{2, \mathbb{C}}\right)^{g}\right)$ par $:$

$\tau \in\left(\mathfrak{H}^{ \pm}\right)^{g} \mapsto\left[\left(z_{1}, z_{2}\right) \in \mathbb{S}(\mathbb{C}) \mapsto\left(\frac{i}{2 \operatorname{Im}\left(\tau_{k}\right)}\left(\begin{array}{cc}\overline{\tau_{k}} z_{1}-\tau_{k} z_{2} & -\left|\tau_{k}\right|^{2}\left(z_{1}-z_{2}\right) \\ z_{1}-z_{2} & -\tau_{k} z_{1}+\overline{\tau_{k}} z_{2}\end{array}\right)\right)_{1 \leq k \leq g} \in G L_{2}(\mathbb{C})^{g}\right]$.

Alors, $\left(G,\left(\mathfrak{H}^{ \pm}\right)^{g}\right)$ est une donnée de Shimura pure. Pour tout $\mathfrak{p}$ ideal premier de $L$, on pose

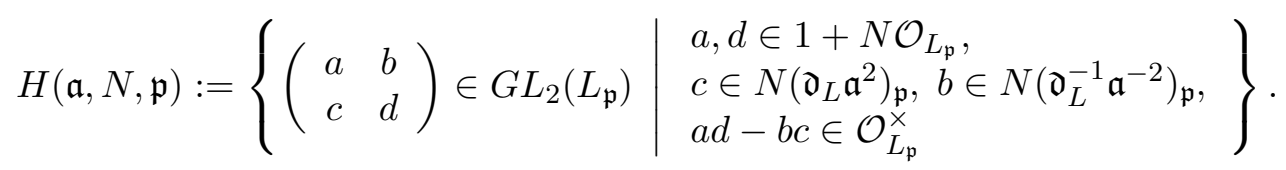

Le groupe $H(\mathfrak{a}, N):=\left(\prod_{\mathfrak{p}} H(\mathfrak{a}, N, \mathfrak{p})\right) \cap G\left(\mathbb{A}_{\mathbb{Q}, f}\right)$ est un sous-groupe compact ouvert net de $G\left(\mathbb{A}_{\mathbb{Q}, f}\right)$.

On note $S$ la variété algébrique complexe quasi-projective lisse $M^{H(\mathfrak{a}, N)}\left(G,\left(\mathfrak{H}^{ \pm}\right)^{g}\right)$ (cf. [G, p. 103] pour une interprétation modulaire de $S$ ). L'inclusion canonique du produit de $g$ copies du demi-plan de Poincaré supérieur $\mathfrak{H}$ dans $\left(\mathfrak{H}^{ \pm}\right)^{g}$ induit une immersion ouverte

$$
\Gamma(\mathfrak{a}, N) \backslash \mathfrak{H}^{g} \hookrightarrow S^{a n},
$$

où $\Gamma(\mathfrak{a}, N):=\left\{\left(\begin{array}{ll}a & b \\ c & d\end{array}\right) \in S L_{2}(L) \mid a, d \in 1+N \mathcal{O}_{L}, c \in N \mathfrak{d}_{L} \mathfrak{a}^{2}, b \in N \mathfrak{d}_{L}^{-1} \mathfrak{a}^{-2}\right\} \subseteq G(\mathbb{Q})$, qui identifie $\Gamma(\mathfrak{a}, N) \backslash \mathfrak{H}^{g}$ à une composante connexe de $S^{a n}$. La variété analytique complexe $\Gamma(\mathfrak{a}, N) \backslash \mathfrak{H}^{g}$ est la variété analytique complexe associée à une variété algébrique complexe quasi-projective lisse canonique notée $S^{0}$. Par définition de la structure de variété algébrique de $S, S^{0}$ est une composante connexe de $S$.

\subsection{Définition de la famille de variétés abéliennes}

Soit $V:=\operatorname{Res}_{L / \mathbb{Q}}\left(\mathbb{G}_{a, L} \oplus \mathbb{G}_{a, L}\right)$. On pose $P:=V \rtimes G$ le produit semi-direct associé à la restriction à $G$ de l'action standard de $\operatorname{Res}_{L / \mathbb{Q}} G L_{2, L}$ sur $V$.

Lemme 2.2 (comparer à [Wi1-V-Lemma 1.1]) - Soient $\tau \in\left(\mathfrak{H}^{ \pm}\right)^{g}$. Le morphisme $h(\tau)$ définit une $\mathbb{Q}$-structure de Hodge pure de poids -1 sur $V(\mathbb{Q})$. Sous l'identification $V_{\mathbb{C}}=\left(\mathbb{G}_{a, \mathbb{C}} \oplus \mathbb{G}_{a, \mathbb{C}}\right)^{g}$ la filtration de Hodge est donnée par :

$$
\begin{aligned}
& F^{1}\left(V_{\mathbb{C}}\right)=0, \\
& F^{0}\left(V_{\mathbb{C}}\right)=\left\langle\left(\begin{array}{c}
\tau_{1} \\
1
\end{array}\right)\right\rangle_{\mathbb{C}} \times \cdots \times\left\langle\left(\begin{array}{c}
\tau_{g} \\
1
\end{array}\right)\right\rangle_{\mathbb{C}}, \\
& F^{-1}\left(V_{\mathbb{C}}\right)=V_{\mathbb{C}} .
\end{aligned}
$$


Démonstration - $\quad H^{0,-1}\left(V_{\mathbb{C}}\right)$ est le sous-espace propre associé au caractère $\left(z_{1}, z_{2}\right) \mapsto z_{2}$. Un calcul permet alors de conclure.

On définit alors la donnée de Shimura $(P, \mathfrak{X})$ comme étant l'extension unipotente de $\left(G,\left(\mathfrak{H}^{ \pm}\right)^{g}\right)$ par $V$. Comme $\operatorname{Lie}(V)$ est de type $\{(-1,0),(0,-1)\}$, on a la description suivante de $\mathfrak{X}$ :

$$
\mathfrak{X}=\left\{(\psi, \tau) \in \operatorname{Hom}\left(\mathbb{S}, P_{\mathbb{R}}\right) \times\left(\mathfrak{H}^{ \pm}\right)^{g} \mid h(\tau)=p \circ \psi\right\},
$$

où $p: P \rightarrow G$ est la projection canonique. À l'aide des isomorphismes donnés par [Wi1-V-Lemma 1.2] et [Wi1-V-Corollary 1.4.b], on construit un biholomorphisme :

$$
\mathbb{C}^{g} \times\left(\mathfrak{H}^{ \pm}\right)^{g} \stackrel{\sim}{\rightarrow} \mathfrak{X}
$$

On introduit le sous-groupe ouvert compact de $P\left(\mathbb{A}_{\mathbb{Q}, f}\right)$

$$
K(\mathfrak{a}, N):=\left(\prod_{\mathfrak{p}}\left(\mathfrak{a}^{\vee}\right)_{\mathfrak{p}} \oplus \prod_{\mathfrak{p}} \mathfrak{a}_{\mathfrak{p}}\right) \rtimes H(\mathfrak{a}, N) .
$$

On note $A$ la variété algébrique complexe quasi-projective lisse $M^{K(\mathfrak{a}, N)}(P, \mathfrak{X})$. La projection canonique $p: P \rightarrow G$ induit un morphisme $A \rightarrow S$, noté (abusivement) également $p$, qui est le morphisme structural d'un schéma abélien de dimension relative pure $g$.

La restriction de $p^{a n}: A^{a n} \rightarrow S^{a n}$ au dessus $\left(S^{0}\right)^{a n}=\Gamma(\mathfrak{a}, N) \backslash \mathfrak{H}^{g}$ est donnée par la projection canonique

$$
q: A^{0}:=\Lambda(\mathfrak{a}, N) \backslash \mathfrak{X}^{+} \rightarrow\left(S^{0}\right)^{a n},
$$

où $\mathfrak{X}^{+}:=\mathbb{C}^{g} \times \mathfrak{H}^{g}$ et $\Lambda(\mathfrak{a}, N):=\left(\mathfrak{a}^{\vee} \oplus \mathfrak{a}\right) \rtimes \Gamma(\mathfrak{a}, N)$ agit sur $\mathfrak{X}^{+}$par l'action qui à

$$
\left(\left(\left(a^{\prime}, a\right),\left(\begin{array}{cc}
\alpha & \beta \\
\gamma & \delta
\end{array}\right)\right),(z, \tau)\right) \in \Lambda(\mathfrak{a}, N) \times\left(\mathbb{C}^{g} \times \mathfrak{H}^{g}\right)
$$

fait correspondre :

$$
\left(\frac{z_{k}}{\sigma_{k}(\gamma) \tau_{k}+\sigma_{k}(\delta)}+\sigma_{k}\left(a^{\prime}\right)-\sigma_{k}(a)\left(\frac{\sigma_{k}(\alpha) \tau_{k}+\sigma_{k}(\beta)}{\sigma_{k}(\gamma) \tau_{k}+\sigma_{k}(\delta)}\right), \frac{\sigma_{k}(\alpha) \tau_{k}+\sigma_{k}(\beta)}{\sigma_{k}(\gamma) \tau_{k}+\sigma_{k}(\delta)}\right)_{1 \leq k \leq g} \in \mathbb{C}^{g} \times \mathfrak{H}^{g}
$$

On a donc l'identification suivante $:\left(p_{\mid S^{0}}: A_{\mid S^{0}} \rightarrow S^{0}\right)^{a n}=\left(q: A^{0} \rightarrow\left(S^{0}\right)^{a n}\right)$.

\section{$2.3 \quad$ La pointe $\infty$}

On rappelle succinctement comment est construite la compactification de Baily-Borel de $S^{0}$. On définit une pointe de $\Gamma(\mathfrak{a}, N)$ comme étant une orbite d'un point de $\mathbb{P}^{1}(L)$ sous l'action de $\Gamma(\mathfrak{a}, N) \subseteq S L_{2}(L)$ induite par les homographies. On adjoint à $S^{0}$ l'ensemble des pointes de $\Gamma(\mathfrak{a}, N)$ pour obtenir un ensemble que l'on munit de la topologie de Satake. Sur cet espace topologique, on dispose d'une structure d'espace analytique complexe normal compact (non lisse si $L \neq \mathbb{Q}$ ) compatible avec celle de $\left(S^{0}\right)^{a n}$. À l'aide des séries de Poincaré, on construit un plongement de cet espace analytique dans un espace projectif. Il hérite ainsi d'une structure de variété algébrique complexe normale et projective. On note $\left(S^{0}\right)^{*}$ la variété algébrique obtenue. Par définition même de la structure algébrique de $S^{0}, S^{0}$ est un ouvert dense de $\left(S^{0}\right)^{*}$ et la variété $\left(S^{0}\right)^{*}$ est appelée compactification de Baily-Borel de $S^{0}$. 
On décrit maintenant un système fondamental de voisinages dans $\left(S^{0}\right)^{*}$ (pour la structure analytique) de la pointe $\infty$, pointe qui correspond à l'orbite de $[1: 0] \in \mathbb{P}^{1}(L)$ sous l'action de $\Gamma(\mathfrak{a}, N)$. Le stabilisateur de l' $\infty$ dans $\Gamma(\mathfrak{a}, N)$ est

$$
\Gamma(\mathfrak{a}, N, \infty):=\left\{\left(\begin{array}{cc}
\varepsilon & a \\
0 & \varepsilon^{-1}
\end{array}\right) \in \Gamma(\mathfrak{a}, N) \mid \varepsilon \in U_{L, N} \text { et } a \in N \mathfrak{d}_{L}^{-1} \mathfrak{a}^{-2}\right\},
$$

où $U_{L, N}$ est le sous-groupe des unités de $\mathcal{O}_{L}$ congrues à 1 modulo $N$. On vérifie que $\Gamma(\mathfrak{a}, N, \infty)$ agit sur

$$
V_{r}:=\left\{\begin{array}{l|l}
\tau \in \mathfrak{H}^{g} & \prod_{i=1}^{g} \operatorname{Im}\left(\tau_{j}\right)>r
\end{array}\right\},
$$

pour tout $r \in \mathbb{R}^{>0}$. Alors pour $r \gg 0$, le morphisme canonique $\Gamma(\mathfrak{a}, N, \infty) \backslash V_{r} \rightarrow S^{0}$ est une immersion ouverte et l'ensemble $\left\{\left(\Gamma(\mathfrak{a}, N, \infty) \backslash V_{r}\right) \cup\{\infty\} \mid r \gg 0\right\}$ forme une base de voisinages ouverts de l' $\infty$ dans $S^{0}$.

\subsection{Sections de torsion}

Soient $a^{\prime} \in N^{-1} \mathfrak{a}^{\vee}, a \in N^{-1} \mathfrak{a}$. Le morphisme analytique

$$
\begin{aligned}
x_{a^{\prime}, a}: \quad\left(S^{0}\right)^{a n}=\Gamma(\mathfrak{a}, N) \backslash \mathfrak{H}^{g} & \rightarrow \Lambda(\mathfrak{a}, N) \backslash\left(\mathbb{C}^{g} \times \mathfrak{H}^{g}\right) \simeq\left(A_{\mid S^{0}}\right)^{a n} \\
{[\tau] } & \mapsto\left[\left(\sigma_{k}\left(a^{\prime}\right)+\sigma_{k}(a) \tau_{k}\right)_{1 \leq k \leq g}, \tau\right] .
\end{aligned}
$$

est le morphisme analytique associé à un unique morphisme algébrique $S^{0} \rightarrow A_{\mid S^{0}}$ qui est une section de $N$-torsion de $p_{\mid S^{0}}: A_{\mid S^{0}} \rightarrow S^{0}$ que l'on note (abusivement) également $x_{a^{\prime}, a}$.

\section{Le morphisme résidu en l' $\infty$}

Cette article traite de la dégénérescence des classes d'Eisenstein (cf. [B2, Définition 5.3]) du schéma abélien $p_{\mid S^{0}}: A_{\mid S^{0}} \rightarrow S^{0}$ en la pointe $\infty$, i.e. de la détermination du résidu en l' $\infty$ des classes d'Eisenstein.

Dans cette partie, on définit le morphisme résidu en l' $\infty$, de source un groupe d'extensions de $M H M\left(S^{0}\right)$ et de but un groupe d'extensions de $S H M$ qui est soit trivial, soit canoniquement isomorphe à $\mathbb{Q}$. On explique enfin que le calcul du résidu en l' $\infty$ se réduit à un calcul au niveau topologique.

On note :

$$
\begin{array}{cl}
j: S^{0} \hookrightarrow\left(S^{0}\right)^{*} & \text { l'immersion ouverte de } S^{0} \text { dans }\left(S^{0}\right)^{*}, \\
i_{\infty}: \infty \hookrightarrow\left(S^{0}\right)^{*} & \text { l'immersion fermée de la pointe } \infty \text { dans }\left(S^{0}\right)^{*}, \\
\mathcal{H} & :=\left(R^{1}\left(p_{\mid S^{0}}\right)_{*} \mathbb{Q}\right)^{\vee} \in \operatorname{Ob}\left(\operatorname{VSH} M\left(S^{0}\right)\right), \\
l & \text { un entier positif. }
\end{array}
$$




\subsection{Définition du morphisme résidu en l' $\infty$}

On définit le morphisme $\operatorname{Res}_{\infty}^{l}$ par le diagramme commutatif suivant.

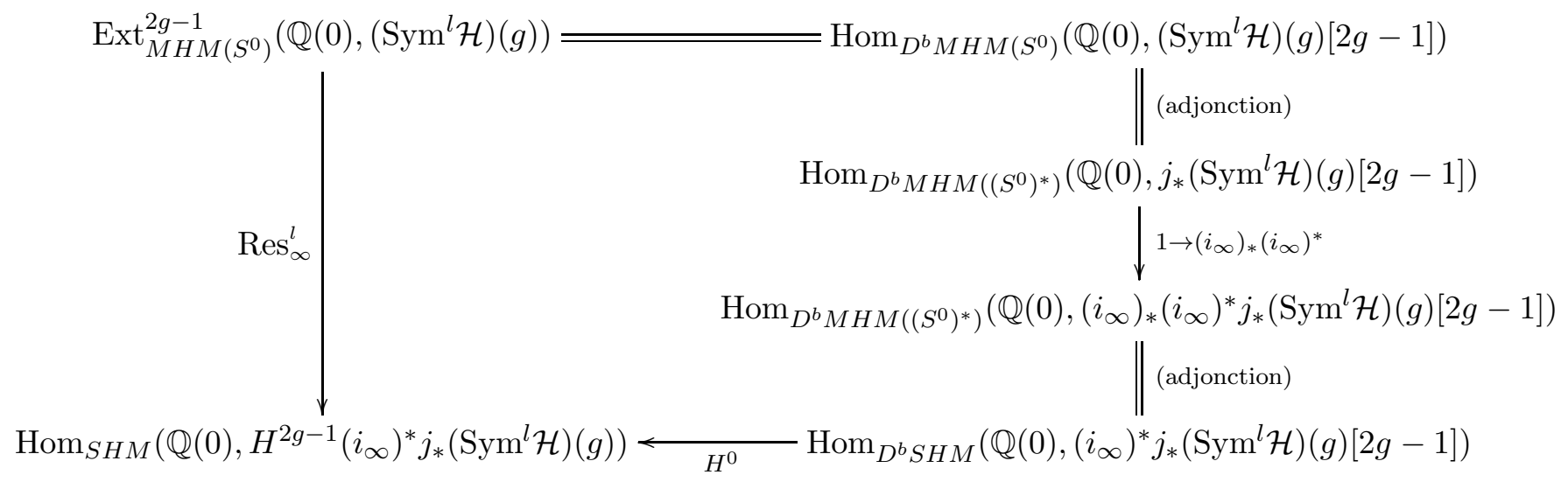

\subsection{Le but du morphisme résidu en l' $\infty$}

On détermine à présent la $\mathbb{Q}$-structure de Hodge $H^{2 g-1}\left(i_{\infty}\right)^{*} j_{*}\left(\operatorname{Sym}^{l} \mathcal{H}\right)(g)$.

Par construction de $S=M^{H(\mathfrak{a}, N)}\left(G,\left(\mathfrak{H}^{ \pm}\right)^{g}\right)$, on dispose d'un foncteur canonique qui associe à une représentation algébrique rationnelle de $G$ une $\mathbb{Q}$-variation de structures de Hodge mixtes polarisable sur $M^{H(\mathfrak{a}, N)}\left(G,\left(\mathfrak{H}^{ \pm}\right)^{g}\right)(\mathbb{C})$. De plus, d'après [W], II - Theorem 2.2], les variations issues de cette construction sont admissibles. On en déduit un foncteur tensoriel canonique

$$
\mu_{H(\mathfrak{a}, N)}: \operatorname{Rep}_{\mathbb{Q}} G \rightarrow \operatorname{MHM}(S),
$$

où $\operatorname{Rep}_{\mathbb{Q}} G$ est la catégorie des représentations algébriques rationnelles de $G$.

D'après la définition de $G$, on a un morphisme canonique $G \rightarrow \mathbb{G}_{m, \mathbb{Q}}$, noté $\chi$, qui fournit une action de $G$ sur $\mathbb{G}_{a, \mathbb{Q}}$. On vérifie que $\mu_{K(\mathfrak{a}, N)}(\chi)=\mathbb{Q}(1)$. Le groupe $G$ agit sur $V:=$ $\operatorname{Res}_{L / \mathbb{Q}}\left(\mathbb{G}_{a, L} \oplus \mathbb{G}_{a, L}\right)$ par restriction de l'action standard de $\operatorname{Res}_{L / \mathbb{Q}} G L_{2, L}$. L'image par $\mu_{H(\mathfrak{a}, N)}$ de cette représentation est $\left(R^{1} p_{*} \mathbb{Q}\right)^{\vee}$ ( $p$ est le morphisme structural du schéma abélien $A \rightarrow S$ ). Ainsi $\left(\operatorname{Sym}^{l}\left(R^{1} p_{*} \mathbb{Q}\right)^{\vee}\right)(g)$ est dans l'image du foncteur $\mu_{H(\mathfrak{a}, N)}$.

Burgos et Wildeshaus ont établi un Théorème [BW, Theorem 2.9] qui permet de calculer la $\mathbb{Q}$-structure de Hodge $H^{2 g-1} i_{\infty}^{*} j_{*} \mu_{H(\mathfrak{a}, N)}(W)$, pour $W \in \operatorname{Rep}_{\mathbb{Q}} G$, en termes de cohomologies de deux groupes : l'un algébrique unipotent, l'autre arithmétique. L'énoncé du résultat précis requiert la description de la compactification de Baily-Borel dans le formalisme de Pink. Pour cela, on renvoie aux chapitres 4 et 6 de la Thèse de Pink $[\mathrm{P}]$ ou encore, pour un résumé, à [BW-p. 365 et 366]. On explicite les différents objets qui interviennent dans la description de la pointe $\infty$ de $B$ en suivant les notations de [BW- p. $365-367]$ et aussi les groupes qui interviennent dans ce cas dans le Théorème [BW-Theorem 2.9].

- Soit $Q$ le sous-groupe parabolique admissible de $G$ qui est le produit fibré du sous-groupe de Borel standard de $\operatorname{Res}_{L / \mathbb{Q}} G L_{2, L}$

$$
\left\{\left(\begin{array}{ll}
* & * \\
0 & *
\end{array}\right)\right\}
$$


et de $\mathbb{G}_{m, \mathbb{Q}}$.

- Le sous-groupe normal canonique de $Q, P_{1}$, est le produit fibré du sous-groupe de $\operatorname{Res}_{L / \mathbb{Q}} G L_{2, L}$

$$
\left\{\left(\begin{array}{ll}
* & * \\
0 & 1
\end{array}\right)\right\}
$$

et de $\mathbb{G}_{m, \mathbb{Q}}$ qui s'identifie à $\operatorname{Res}_{L / \mathbb{Q}} \mathbb{G}_{a, L} \rtimes \mathbb{G}_{m, \mathbb{Q}}$.

- Le radical unipotent $W_{1}$ de $P_{1}$ (et de $Q$ ) est le produit fibré du sous-groupe de $\operatorname{Res}_{L / \mathbb{Q}} G L_{2, L}$

$$
\left\{\left(\begin{array}{ll}
1 & * \\
0 & 1
\end{array}\right)\right\}
$$

et de $\mathbb{G}_{m, \mathbb{Q}}$, c'est à dire $\operatorname{Res}_{L / \mathbb{Q}} \mathbb{G}_{a, L}$.

- On note $\pi: P_{1} \rightarrow G_{1}:=P_{1} / W_{1}=\mathbb{G}_{m, \mathbb{Q}}$ la projection canonique.

- On fixe $K:=H(\mathfrak{a}, N)$ sous-groupe compact ouvert net de $G\left(\mathbb{A}_{\mathbb{Q}, f}\right), g:=1 \in G\left(\mathbb{A}_{\mathbb{Q}, f}\right)$ et on pose $K_{1}:=P_{1}\left(\mathbb{A}_{\mathbb{Q}, f}\right) \cap H(\mathfrak{a}, N)$.

- La composante de bord dans la compactification de Baily-Borel qui correspond à la pointe $\infty$ est la donnée de Shimura $\left(\mathbb{G}_{m, \mathbb{Q}}, \operatorname{Isom}(\mathbb{Z}, \mathbb{Z}(1))\right.$ ) (cf. $[\mathbb{P}$, Example 2.8]).

- On a donc un morphisme $i_{\mathbb{G}_{m, \mathbb{Q}}, H(\mathfrak{a}, N), 1}: M^{\pi\left(K_{1}\right)}\left(\mathbb{G}_{m, \mathbb{Q}}, \operatorname{Isom}(\mathbb{Z}, \mathbb{Z}(1))\right) \rightarrow\left(S^{0}\right)^{*}$.

- On calcule $H_{C}:=\operatorname{Cent}_{Q(\mathbb{Q})}(\{-1,1\}) \cap W_{1}\left(\mathbb{A}_{\mathbb{Q}, f}\right) \cdot H(\mathfrak{a}, N)$ et $\overline{H_{C}}:=\pi\left(H_{C}\right)$ pour trouver

$$
H_{C}=\left\{\left(\begin{array}{cc}
\varepsilon & * \\
0 & \varepsilon^{-1}
\end{array}\right) \mid \varepsilon \in U_{L, N}\right\} \quad \text { et } \quad \overline{H_{C}}=\left\{\left[\left(\begin{array}{cc}
\varepsilon & * \\
0 & \varepsilon^{-1}
\end{array}\right)\right] \mid \varepsilon \in U_{L, N}\right\} \simeq U_{L, N} .
$$

- Enfin, $\Delta \backslash M^{\pi\left(K_{1}\right)}\left(\mathbb{G}_{m, \mathbb{Q}}, \operatorname{Isom}(\mathbb{Z}, \mathbb{Z}(1))\right)$ (cf. [BW, p. 367] pour la définition de $\Delta$ ) est réduit à un point et l'immersion induite par $i_{\mathbb{G}_{m, \mathbb{Q}}, H(\mathfrak{a}, N), 1}: M^{\pi\left(K_{1}\right)}\left(\mathbb{G}_{m, \mathbb{Q}}, \operatorname{Isom}(\mathbb{Z}, \mathbb{Z}(1))\right) \rightarrow\left(S^{0}\right)^{*}$

$$
\Delta \backslash M^{\pi\left(K_{1}\right)}\left(\mathbb{G}_{m, \mathbb{Q}}, \operatorname{Isom}(\mathbb{Z}, \mathbb{Z}(1))\right) \hookrightarrow\left(S^{0}\right)^{*}
$$

correspond à $i_{\infty}: \infty \hookrightarrow\left(S^{0}\right)^{*}$.

On peut alors énoncer le résultat suivant.

Théorème 3.1 (cas particulier de [BW-Theorem 2.9]) -

$$
H^{2 g-1} i_{\infty}^{*} j_{*}\left(\operatorname{Sym}^{l} \mathcal{H}\right)(g)=\underset{p+q=2 g-1}{\oplus} \mu_{\pi\left(K_{1}\right)} \circ H^{p}\left(\overline{H_{C}}, H^{q}\left(W_{1}, \operatorname{Res}_{Q}^{G}\left(\left(\operatorname{Sym}^{l} V\right) \otimes \chi^{g}\right)\right)\right) .
$$

La dimension cohomologique du groupe $W_{1}$ (resp. du groupe abélien libre de rang $g-1$ sans torsion $\overline{H_{C}}$ ) est $g$ (resp. $g-1$ ). Ainsi, on a :

$$
H^{2 g-1} i_{\infty}^{*} j_{*}\left(\operatorname{Sym}^{l} \mathcal{H}\right)(d)=\mu_{\pi\left(K_{1}\right)} \circ H^{2 g-1}\left(\overline{H_{C}}, H^{g}\left(W_{1}, \operatorname{Res}_{Q}^{G}\left(\left(\operatorname{Sym}^{l} V\right) \otimes \chi^{g}\right)\right)\right) .
$$

\section{Proposition $3.2-$}

$$
H^{2 g-1} i_{\infty}^{*} j_{*}\left(\operatorname{Sym}^{l} \mathcal{H}\right)(g)=\left\{\begin{array}{cl}
\mathbb{Q}(0) & \text { sig divise } l \\
0 & \text { sinon }
\end{array}\right.
$$


a) Calcul de $H^{g}\left(W_{1}, \operatorname{Res}_{Q}^{G}\left(\operatorname{Sym}^{l} V \otimes \chi^{g}\right)\right)$

On commence par remarquer que $W_{1}$ n'agit pas sur $\chi^{g}$. D'après [Kn-Thm 6.10], on a un isomorphisme $\left(Q / W_{1}\right)$-équivariant :

$$
H^{g}\left(W_{1}, \operatorname{Res}_{Q}^{G}\left(\operatorname{Sym}^{l}\right)\right) \simeq H_{0}\left(W_{1}, \operatorname{Res}_{Q}^{G}\left(\operatorname{Sym}^{l} V\right)\right) \otimes \stackrel{g}{\wedge}\left(\operatorname{Lie} W_{1}\right)^{\vee} .
$$

On étend ensuite les scalaires à $\overline{\mathbb{Q}}$, une clôture algébrique de $\mathbb{Q}$. On a l'isomorphisme canonique suivant :

$$
H_{0}\left(W_{1}, \operatorname{Res}_{Q}^{G}\left(\operatorname{Sym}^{l} V\right)\right) \otimes_{\mathbb{Q}} \overline{\mathbb{Q}}=H_{0}\left(W_{1}(\overline{\mathbb{Q}}), \operatorname{Sym}^{l} V(\overline{\mathbb{Q}})\right) .
$$

On note que :

$$
\begin{aligned}
W_{1}(\overline{\mathbb{Q}}) & =\prod_{i=1}^{g}\left\{\left(\begin{array}{cc}
1 & \alpha \\
0 & 1
\end{array}\right) \mid \alpha \in \overline{\mathbb{Q}}\right\} \\
V(\overline{\mathbb{Q}}) & =\prod_{i=1}^{g}\left\{a_{i} X_{i}+b_{i} Y_{i} \mid a_{i}, b_{i} \in \overline{\mathbb{Q}}\right\},
\end{aligned}
$$

où $X_{i}=(1,0), Y_{i}=(0,1) \in \overline{\mathbb{Q}}^{2}$. On a ainsi une base canonique pour $\operatorname{Sym}^{l} V(\overline{\mathbb{Q}})$ :

$$
\left(X_{1}^{m_{1}} \ldots X_{g}^{m_{g}} Y_{1}^{n_{1}} \ldots Y_{g}^{n_{g}}\right)_{\left(m_{1}, \ldots, m_{g}, n_{1}, \ldots, n_{g}\right)}
$$

indexée par les $2 g$-uplets d'entiers positifs $\left(m_{1}, \ldots, m_{g}, n_{1}, \ldots, n_{g}\right)$ tels que :

$$
m_{1}+\cdots+m_{g}+n_{1}+\cdots+n_{g}=l .
$$

Soit $\alpha \in \overline{\mathbb{Q}}$ et soit $w:=\left(\begin{array}{ll}1 & \alpha \\ 0 & 1\end{array}\right)$. Alors, $w \cdot X_{i}=X_{i}$ et $w Y_{i}=\alpha X_{i}+Y_{i}$ et on vérifie, à l'aide de cette remarque, que :

$$
H_{0}\left(W_{1}(\overline{\mathbb{Q}}), \operatorname{Sym}^{l} V(\overline{\mathbb{Q}})\right)=\operatorname{Sym}^{l} V(\overline{\mathbb{Q}}) /\left\langle\left\{X_{1}^{m_{1}} \ldots X_{g}^{m_{g}} Y_{1}^{n_{1}} \ldots Y_{g}^{n_{g}} \mid \exists i \text { tel que } m_{i} \neq 0\right\}\right\rangle_{\overline{\mathbb{Q}}} .
$$

b) Calcul de $H^{g-1}\left(\overline{H_{C}},\left(\operatorname{Sym}^{l} V(\overline{\mathbb{Q}}) /\left\langle\left\{X_{1}^{m_{1}} \ldots X_{g}^{m_{g}} Y_{1}^{n_{1}} \ldots Y_{g}^{n_{g}} \mid \exists i \text { tel que } m_{i} \neq 0\right\}\right\rangle_{\overline{\mathbb{Q}}}\right) \otimes \chi^{g}\right)$.

Tout d'abord, $\overline{H_{C}}$ n'agit ni sur $\chi^{g}$ (les éléments de $\overline{H_{C}}$ sont de déterminant 1 ) ni sur $\stackrel{g}{\wedge}\left(\mathrm{Lie}_{1}\right)^{\vee}$ (le carré d'une unité est de norme 1$)$. Le groupe $\overline{H_{C}}$ étant isomorphe à $\mathbb{Z}^{g-1}$, en prenant une résolution de Koszul, on obtient un isomorphisme entre :

$$
H^{g-1}\left(\overline{H_{C}},\left(\operatorname{Sym}^{l} V(\overline{\mathbb{Q}}) /\left\langle\left\{X_{1}^{m_{1}} \ldots X_{g}^{m_{g}} Y_{1}^{n_{1}} . . Y_{g}^{n_{g}} \mid \exists i \text { tel que } m_{i} \neq 0\right\}\right\rangle_{\overline{\mathbb{Q}}}\right)\right)
$$

et

$$
H_{0}\left(\overline{H_{C}},\left(\operatorname{Sym}^{l} V(\overline{\mathbb{Q}}) /\left\langle\left\{X_{1}^{m_{1}} \ldots X_{g}^{m_{g}} Y_{1}^{n_{1}} \ldots Y_{g}^{n_{g}} \mid \exists i \text { tel que } m_{i} \neq 0\right\}\right\rangle_{\overline{\mathbb{Q}}}\right) .\right.
$$

Soient $\left(n_{1}, \ldots, n_{g}\right)$ un $g$-uplet d'entiers positifs tels que $n_{1}+\cdots+n_{g}=l$ et

$$
h=\left[\left(\begin{array}{cc}
\varepsilon & * \\
0 & \varepsilon^{-1}
\end{array}\right)\right] \in \overline{H_{C}}
$$


alors, $h .\left[Y_{1}^{n_{1}} \ldots Y_{g}^{n_{g}}\right]=\left[\sigma_{1}\left(\varepsilon^{-1}\right)^{n_{1}} \ldots \sigma_{g}\left(\varepsilon^{-1}\right)^{n_{g}} Y_{1}^{n_{1}} \ldots Y_{g}^{n_{g}}\right]$. On remarque que si les $n_{i}$ ne sont pas tous égaux, alors il existe $\varepsilon \in U_{L, N}$ tel que $\sigma_{1}\left(\varepsilon^{-1}\right)^{n_{1}} \ldots \sigma_{g}\left(\varepsilon^{-1}\right)^{n_{g}} \neq 1$. Ceci peut se voir en utilisant une $\mathbb{Z}$-base $\left(u_{1}, \ldots, u_{g-1}\right)$ de $U_{L, N}$ et le résultat de théorie des nombres classique qui affirme que :

$$
\operatorname{det}\left(\begin{array}{ccc}
\log \left|\sigma_{1}\left(u_{1}\right)\right| & \ldots & \log \left|\sigma_{g-1}\left(u_{1}\right)\right| \\
\vdots & \ldots & \vdots \\
\log \left|\sigma_{1}\left(u_{g-1}\right)\right| & \ldots & \log \left|\sigma_{g-1}\left(u_{g-1}\right)\right|
\end{array}\right) \neq 0 .
$$

c) Conclusion

On rassemble les résultats précédents. On définit $W$ comme suit.

- Cas où $g$ divise $l:$ On pose $\lambda:=l / g \in \mathbb{N}$ et on définit $W$ comme étant le sous-espace de $\operatorname{Sym}^{l} V(\overline{\mathbb{Q}})$ engendré par les $X_{1}^{m_{1}} \ldots X_{g}^{m_{g}} Y_{1}^{n_{1}} \ldots Y_{g}^{n_{g}}$ tels que $\left(n_{1}, \ldots, n_{g}\right) \neq(\lambda, \ldots, \lambda)$.

- Cas où $g$ ne divise pas $l: \quad$ On pose $W:=\operatorname{Sym}^{l} V(\overline{\mathbb{Q}})$.

On a prouvé que :

$$
H^{2 g-1} i_{\infty}^{*} j_{*}\left(\operatorname{Sym}^{l} \mathcal{H}\right)(d) \otimes \mathbb{Q} \overline{\mathbb{Q}}=\operatorname{Sym}^{l} V(\overline{\mathbb{Q}}) / W \otimes \stackrel{g}{\wedge}\left(\operatorname{Lie} W_{1}\right)^{\vee} \otimes \chi^{g} .
$$

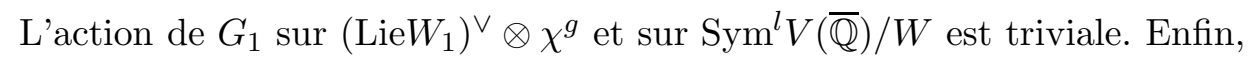

$$
\operatorname{dim}_{\overline{\mathbb{Q}}} \operatorname{Sym}^{l} V(\overline{\mathbb{Q}}) / W=\left\{\begin{array}{l}
1 \text { si } g \text { divise } l, \\
0 \text { sinon. }
\end{array}\right.
$$

Remarque 3.3 - Sig divise l, alors le quotient $\operatorname{Sym}^{l} V(\overline{\mathbb{Q}}) / W$ (engendré par la classe $\left[Y_{1}^{l / g} \ldots Y_{g}^{l / g}\right]$ ) et la projection canonique $\operatorname{Sym}^{l} V(\overline{\mathbb{Q}}) \rightarrow \operatorname{Sym}^{l} V(\overline{\mathbb{Q}}) / W$ sont définis sur $\mathbb{Q}$.

\subsection{Rigidité de la dégénerescence}

On suppose ici que $g$ divise $l$. La construction de $\operatorname{Res}_{\infty}^{l}$ admet un analogue évident au niveau topologique (i.e. dans la théorie $\left.D_{c}^{b}(\cdot)\right)$ qui permet de définir un morphisme

$$
\overline{\operatorname{Res}_{\infty}^{l}}: H_{\mathrm{Betti}}^{2 g-1}\left(S^{0}(\mathbb{C}), \overline{\left(\operatorname{Sym}^{l} \mathcal{H}\right)(g)}\right) \rightarrow H_{\mathrm{Betti}}^{2 g-1}\left(\infty, i_{\infty}^{*} R j_{*} \overline{\left(\operatorname{Sym}^{l} \mathcal{H}\right)(g)}\right) .
$$

Ce morphisme s'insère dans le diagramme suivant

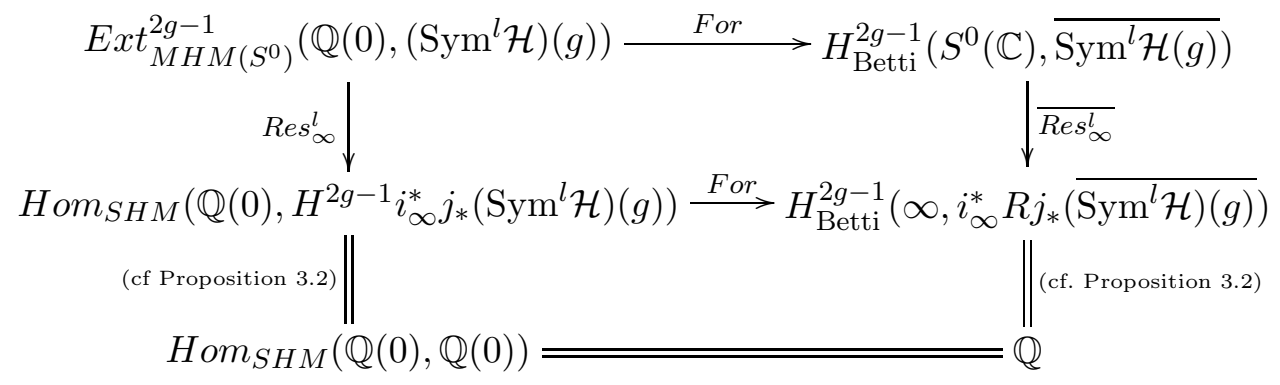

qui est commutatif. En effet, le formalisme des 6 foncteurs de $D^{b} M H M(\cdot)$ et celui de $D_{c}^{b}(\cdot)$ sont compatibles via le foncteur For. On en déduit la Proposition suivante. 
Proposition 3.4 - Pour tout $c \in \operatorname{Ext}_{M H M\left(S^{0}\right)}^{2 g-1}\left(\mathbb{Q}(0),\left(\operatorname{Sym}^{l} \mathcal{H}\right)(g)\right)$,

$$
\operatorname{Res}_{\infty}^{l}(c)=\overline{\operatorname{Res} s_{\infty}^{l}} \circ \operatorname{For}(c) \in \mathbb{Q}
$$

\section{Calcul des classes d'Eisenstein au niveau topologique}

Dans cette partie, on détermine les classes d'Eisenstein (cf. [B2, Partie 5] pour la définition) du schéma abélien $p_{\mid S^{0}}: A_{\mid S^{0}} \rightarrow S^{0}$ au niveau topologique en utilisant le résultat principal de [B2] (cf. [B2, Corollaire 4.7 ]). Ce dernier assure que les courants définis par Levin dans [L] permettent de décrire le polylogarithme de $p_{\mid S^{0}}: A_{\mid S^{0}} \rightarrow S^{0}$ au niveau topologique, et par suite (cf. [B2, Partie $5]$ ) les classes d'Eisenstein, toujours au niveau topologique.

Il s'agit donc ici d'expliciter les courants de Levin dans notre situation géométrique. Ces courants sont des séries de formes différentielles notées $g_{a, \gamma}^{\prime}$ dans $[\mathrm{L}]$. Pour la définition précise de ces formes, on renvoie le lecteur à $[\mathrm{L}$. On en donne ci-dessous une esquisse tout en présentant le contenu de cette quatrième partie.

La construction de Levin vaut pour une famille de tores analytiques complexes munie d'une polarisation (cf. [L, 1.1.2]). Dans la partie 4.1, on définit cette polarisation et on explicite le pullback de

$$
\left(p_{\mid S^{0}}: A_{\mid S^{0}} \rightarrow\left(S^{0}\right)\right)^{a n}=\left(q: \Lambda(\mathfrak{a}, N) \backslash\left(\mathbb{C}^{g} \times \mathfrak{H}^{g}\right) \rightarrow \Gamma(\mathfrak{a}, N) \backslash \mathfrak{H}^{g}\right)
$$

par la projection canonique $\mathfrak{H}^{g} \rightarrow \Lambda(\mathfrak{a}, N) \backslash \mathfrak{H}^{g}$, noté $q^{\prime}: A^{\prime} \rightarrow \mathfrak{H}^{g}$. Pour la famille de tores analytiques complexes $q^{\prime}: A^{\prime} \rightarrow \mathfrak{H}^{g}$, on dispose de coordonnées globales pour expliciter $g_{a, \gamma}^{\prime}$. C'est la raison pour laquelle on considère plutot cette famille que $\left(p_{\mid S^{0}}: A_{\mid S^{0}} \rightarrow\left(S^{0}\right)\right)^{a n}$.

On donne alors une trivialisation $\mathcal{C}^{\infty}$-réelle $\Psi$ de la famille de tores réels $q^{\prime}: A^{\prime} \rightarrow \mathfrak{H}^{g}$

$$
\Psi:\left(\Pi_{\mathbb{R}} / \Pi\right) \times \mathfrak{H}^{g} \rightarrow A^{\prime},
$$

où $\Pi:=\mathfrak{a}^{\vee} \oplus \mathfrak{a}$. Le système local constant $\left(R^{1} q_{*}^{\prime} \mathbb{Z}\right)^{\vee}$ s'identifie à $\Pi$ et la polarisation (principale) est donnée par un accouplement $<\cdot, \cdot>: \Pi \wedge \Pi \rightarrow 2 \pi i \mathbb{Z}$. En outre, $\left(R^{1} q_{*}^{\prime} \mathbb{Z}\right)^{\vee}$ est muni d'une structure de variation de structures de Hodge pure de poids -1 . On note $E$ le fibré holomorphe sur $\mathfrak{H}^{g}$ associé et $E=E^{-1,0} \oplus E^{0,-1}$ la décomposition de Hodge de celui-ci. À l'aide de la trivialisation $\Psi$, on associe à toute section $\theta$ de $E$ un champ de vecteurs sur $A^{\prime}$ que l'on note également $\theta$. En particulier, pour tout $\gamma \in \Pi$, on obtient deux champs de vecteurs $\gamma^{-1,0}$ et $\gamma^{0,-1}$ sur $A^{\prime}$, où $\gamma=\gamma^{-1,0}+\gamma^{0,-1}$ est la décomposition de Hodge de $\gamma$ (cf. Partie 4.3 pour une expression en coordonnées de $\gamma^{-1,0}$ et $\left.\gamma^{0,-1}\right)$. Toujours grâce à la trivialisation $\Psi$, on construit une décomposition du fibré tangent $\mathcal{C}^{\infty}$-complexe de $A^{\prime}: T_{\mathbb{C}} A^{\prime}=\left(q^{\prime}\right)^{*} T_{\mathbb{C}} \mathfrak{H}^{g} \oplus\left(A^{\prime} \times \Pi_{\mathbb{C}}\right)$ où $T_{\mathbb{C}} \mathfrak{H}^{g}$ désigne le fibré tangent $\mathcal{C}^{\infty}$-complexe de $\mathfrak{H}^{g}$. La projection canonique $T_{\mathbb{C}} A^{\prime} \rightarrow A^{\prime} \times \Pi_{\mathbb{C}}$ donne une forme différentielle $\nu$ sur $A^{\prime}$ à valeurs dans le fibré $A^{\prime} \times \Pi_{\mathbb{C}}$ (cf. Partie 4.4 pour une expression de $\nu$ en coordonnées). Pour $a \in \mathbb{N} \geq 1$ et $\gamma \in \Pi \backslash\{0\}$, la forme différentielle $g_{a, \gamma}^{\prime} \in \Gamma\left(A^{\prime}, \Omega_{A^{\prime}}^{2 g-1} \otimes \operatorname{Sym}^{a-1} E\right)$ est définie par

$$
g_{a, \gamma}^{\prime}=i_{\gamma^{0,-1}}\left(\chi_{\gamma} \times \frac{1}{\left(\rho(\gamma)-L_{\gamma^{-1,0}}\right)^{a}} \operatorname{vol} \times\left(\gamma^{0,-1}\right)^{a-1}\right),
$$


où - $\quad i_{\gamma^{0,-1}}$ est l'opérateur de contraction par le champ de vecteurs $\gamma^{0,-1}$,

- $\quad \chi_{\gamma}$ est une fonction complexe sur $A^{\prime}$ définie par

$$
\chi_{\gamma}([(z, \tau)])=\exp \left(<\gamma, p r_{1} \circ \Psi^{-1}([(z, \tau)])>\mathbb{C}\right),
$$

$p r_{1}$ désignant la projection canonique $\left(\Pi_{\mathbb{R}} / \Pi\right) \times \mathfrak{H}^{g} \rightarrow \Pi_{\mathbb{R}} / \Pi$ et $<\cdot, \cdot>\mathbb{C}$

l'accouplement obtenu en prolongeant par linéarité $<\cdot, \cdot>$ (cf. Partie 4.6 pour une expression de $\chi_{\gamma}$ en coordonnées),

- $\rho(\gamma)=<\gamma^{-1,0}, \gamma^{0,-1}>_{\mathbb{C}}$ (cf. Partie 4.5 pour une expression de $\rho(\gamma)$ en coordonnées),

- $L_{\gamma^{-1,0}}$ est la dérivée de Lie associée au champ de vecteurs $\gamma^{-1,0}$,

- $v o l=(-1)^{g}(g !)^{-1} \omega^{g}$, où $\omega:=\frac{1}{2}<\nu, \nu>_{\mathbb{C}}$ (cf. Partie 4.4 pour une expression de $\omega$ en coordonnées).

La forme $g_{a, \gamma}^{\prime}$ est bien définie car $L_{\gamma^{-1,0}}^{k} v o l=0$ pour $k>2 g$ (cf. [L, Prop 3.2.2]). Dans la partie 4.7, on effectue le calcul en coordonnées de

$$
i_{\gamma^{0,-1}}\left(\chi_{\gamma} \times \frac{1}{\left(\rho(\gamma)-L_{\gamma^{-1,0}}\right)^{a}} \omega^{g}\right) .
$$

Dans une dernière sous-partie, on applique les résultats de la Partie 5 de [B2], pour obtenir une expression explicite des classes d'Eisenstein dans cette situation géométrique (cf. Prop 4.3).

\subsection{Polarisation et trivialisation $\mathcal{C}^{\infty}$-réelle}

Le calcul des courants de Levin recquiert une polarisation. On précise ici celle que l'on considère dans la suite.

Le pullback de la famille de tores complexes $q: \Lambda^{\prime}(\mathfrak{a}, N) \backslash\left(\mathbb{C}^{g} \times \mathfrak{H}^{g}\right) \rightarrow \Lambda(\mathfrak{a}, N) \backslash \mathfrak{H}^{g}$ par la projection canonique $\mathfrak{H}^{g} \rightarrow \Lambda(\mathfrak{a}, N) \backslash \mathfrak{H}^{g}$ est

$$
q^{\prime}: A^{\prime}:=\Pi \backslash\left(\mathbb{C}^{g} \times \mathfrak{H}^{g}\right) \rightarrow \mathfrak{H}^{g},
$$

où le quotient de $\mathbb{C}^{g} \times \mathfrak{H}^{g}$ par le groupe $\Pi:=\mathfrak{a}^{\vee} \oplus \mathfrak{a}$ est celui associé à l'action

$$
\begin{array}{ccc}
\left(\mathfrak{a}^{\vee} \oplus \mathfrak{a}\right) \times\left(\mathbb{C}^{g} \times \mathfrak{H}^{g}\right) & \rightarrow & \mathbb{C}^{g} \times \mathfrak{H}^{g} \\
\left(\left(a^{\prime}, a\right),(z, \tau)\right) & \mapsto & \left(\left(\sigma_{k}\left(a^{\prime}\right)+\sigma_{k}(a) \tau_{k}+z_{k}\right)_{1 \leq k \leq g}, \tau\right) .
\end{array}
$$

Soit $\tau \in \mathfrak{H}^{g}$. On note $\varphi_{\tau}$ le monomorphisme $\varphi_{\tau}: \Pi \rightarrow \mathbb{C}^{g},\left(a^{\prime}, a\right) \mapsto\left(\left(\sigma_{k}\left(a^{\prime}\right)+\sigma_{k}(a) \tau_{k}\right)_{1 \leq k \leq g}\right.$. Le morphisme $\varphi_{\tau} \otimes \mathbb{R}: \Pi \otimes \mathbb{R} \rightarrow \mathbb{C}^{g}$ déduit de $\varphi_{\tau}$ par linéarité est un isomorphisme de $\mathbb{R}$-vectoriels.

La fibre en $\tau \in \mathfrak{H}^{g}$ de $q^{\prime}$, notée $A_{\tau}^{\prime}$, est $\mathbb{C}^{g} / \varphi_{\tau}(\Pi)$ et l'application

$$
\begin{array}{rlrl}
<\cdot, \cdot>: & \Pi \wedge \Pi & \rightarrow 2 \pi i \mathbb{Z} \\
\left(a_{1}^{\prime}, a_{1}\right) \wedge\left(a_{2}^{\prime}, a_{2}\right) & \mapsto 2 \pi i \operatorname{Tr}_{L}\left(a_{2}^{\prime} a_{1}-a_{1}^{\prime} a_{2}\right)
\end{array}
$$

induit, via $\varphi_{\tau}$, une polarisation principale sur $A_{\tau}^{\prime}$.

On a en outre un isomorphisme $\mathcal{C}^{\infty}$-réel de familles de tores réels au dessus de $\mathfrak{H}^{g}$

$$
\begin{aligned}
\Psi:\left(\Pi_{\mathbb{R}} / \Pi\right) \times \mathfrak{H}^{g} & \rightarrow A^{\prime}=\Pi \backslash\left(\mathbb{C}^{g} \times \mathfrak{H}^{g}\right) . \\
([\xi], \tau) & \mapsto\left[\left(\varphi_{\tau} \otimes \mathbb{R}(\xi), \tau\right)\right]
\end{aligned}
$$


Celui-ci joue un rôle fondamental dans la construction des courants de Levin (cf. [L, Part 2.2] pour la définition de cette trivialisation dans le cas général).

Il est utile pour la suite d'expliciter l'inverse de $\Psi$. On fixe $\left(\alpha_{1}, \ldots, \alpha_{g}\right)$ une $\mathbb{Z}$-base de $\mathfrak{a}$ et on note $\left(\alpha_{1}^{\prime}, \ldots, \alpha_{g}^{\prime}\right)$ la $\mathbb{Z}$-base de $\mathfrak{a}^{\vee}$ duale de $\left(\alpha_{1}, \ldots, \alpha_{g}\right)$ relativement à la forme bilinéaire $\mathfrak{a}^{\vee} \times \mathfrak{a} \rightarrow \mathbb{Z},\left(\alpha^{\prime}, \alpha\right) \mapsto \operatorname{Tr}_{L}\left(\alpha^{\prime} \alpha\right)$.

Notations $4.1-$

- Pour $z=\left(z_{1} \ldots, z_{g}\right) \in \mathbb{C}^{g}$, on note $D(z)$ la matrice diagonale $g \times g$ dont la diagonale est $\left(z_{1}, \ldots, z_{g}\right)$ et $\bar{z}:=\left(\overline{z_{1}}, \ldots, \overline{z_{g}}\right)$.

- On introduit les deux matrices $g \times g$

$$
M:=\left(\sigma_{l}\left(\alpha_{k}\right)\right)_{1 \leq k, l \leq g} \quad \text { et } \quad M^{\prime}:=\left(\sigma_{l}\left(\alpha_{k}^{\prime}\right)\right)_{1 \leq k, l \leq g}
$$

- Pour $\tau=\left(\tau_{1}, \ldots, \tau_{g}\right) \in \mathfrak{H}^{g}$, on pose

$$
T(\tau):=\left(t_{1}, . ., t_{g}\right):=\left(\left(\tau_{1}-\overline{\tau_{1}}\right)^{-1}, \ldots,\left(\tau_{g}-\overline{\tau_{g}}\right)^{-1}\right) .
$$

À l'aide des deux $\mathbb{Z}$-bases $\left(\alpha_{1}, \ldots, \alpha_{g}\right)$ et $\left(\alpha_{1}^{\prime}, \ldots, \alpha_{g}^{\prime}\right)$, on effectue un calcul élémentaire pour montrer que l'image de $[(z, \tau)] \in A^{\prime}=\Pi \backslash\left(\mathbb{C}^{g} \times \mathfrak{H}^{g}\right)$ par le morphisme $\mathcal{C}^{\infty}$-réel $\Psi^{-1}$ est l'élément $\operatorname{de}\left(\Pi_{\mathbb{R}} / \Pi\right) \times \mathfrak{H}^{g}$

$$
\left(\left[(z-\bar{z}) D(T(\tau)) M^{-1}\left(\begin{array}{c}
\alpha_{1} \\
\vdots \\
\alpha_{g}
\end{array}\right)+\left(\bar{z} D(T(\tau)) D(\tau)\left(M^{\prime}\right)^{-1}-z D(T(\tau)) D(\bar{\tau})\left(M^{\prime}\right)^{-1}\right)\left(\begin{array}{c}
\alpha_{1}^{\prime} \\
\vdots \\
\alpha_{g}^{\prime}
\end{array}\right)\right], \tau\right) .
$$

\subsection{Décomposition de Hodge}

On fixe $\tau \in \mathfrak{H}^{g}$. On note $\left(u_{\tau, 1}, \ldots, u_{\tau, g}\right)$ (resp. $\left.\bar{u}_{\tau, 1}, \ldots, \bar{u}_{\tau, g}\right)$ la base de duale de $d z:=$ $\left(d z_{1}, \ldots, d z_{g}\right)$ (resp. $\left.d \bar{z}:=\left(d \overline{z_{1}}, \ldots, d \overline{z_{g}}\right)\right)$ des formes holomorphes (resp. antiholomorphes) de $A_{\tau}^{\prime}$, la fibre de $q^{\prime}$ au dessus de $\tau$.

L'égalité matricielle suivante nous fournit une expression de $u_{\tau, 1}, \ldots, u_{\tau, g}, \bar{u}_{\tau, 1}, \ldots, \bar{u}_{\tau, g}$ dans $\Pi_{\mathbb{C}}=H_{1}\left(A_{\tau}^{\prime}, \mathbb{C}\right)$.

$$
\left(\begin{array}{c}
u_{\tau, 1} \\
\vdots \\
u_{\tau, g} \\
\bar{u}_{\tau, 1} \\
\vdots \\
\bar{u}_{\tau, g}
\end{array}\right)=\left(\begin{array}{cc}
D(T(\tau)) M^{-1} & -D(T(\tau)) D(\bar{\tau})\left(M^{\prime}\right)^{-1} \\
-D(T(\tau)) M^{-1} & D(T(\tau)) D(\tau)\left(M^{\prime}\right)^{-1}
\end{array}\right)\left(\begin{array}{c}
\alpha_{1} \\
\vdots \\
\alpha_{g} \\
\alpha_{1}^{\prime} \\
\vdots \\
\alpha_{g}^{\prime}
\end{array}\right)
$$

On note $E:=E^{-1,0} \oplus E^{0,-1}$ la décomposition de Hodge du fibré vectoriel analytique au dessus de $\mathfrak{H}^{g}$ attachée à la variation de structures de Hodge pures de poids $-1\left(R^{1} q_{*} \mathbb{Z}\right)^{\vee}$. La trivialisation $\mathcal{C}^{\infty}$-réelle $\Psi$ introduite dans la partie 4.1 induit une identification entre $\overline{\left(R^{1} q_{*} \mathbb{Z}\right)^{\vee}}$ (système local 
constant) et $\Pi$.

Soit $\gamma=\left(a^{\prime}, a\right) \in \Pi$. Cet élément définit une section de $q^{*} E$ au dessus de $A^{\prime}$

$$
A^{\prime} \rightarrow A^{\prime} \times \Pi_{\mathbb{C}}, \quad[(z, \tau)] \mapsto([(z, \tau)], \gamma)
$$

que l'on note également (abusivement) $\gamma$. On vérifie que la décomposition de Hodge de $\gamma$ est donnée par :

$$
\begin{aligned}
& \gamma^{-1,0}: \quad A^{\prime} \quad \rightarrow \quad q^{*} E^{-1,0} \\
& {[(z, \tau)] \mapsto\left([(z, \tau)], \sum_{k=1}^{g}\left(\sigma_{k}\left(a^{\prime}\right)+\sigma_{k}(a) \tau_{k}\right) u_{\tau, k}\right)} \\
& \gamma^{0,-1}: \quad A^{\prime} \quad \rightarrow \quad q^{*} E^{0,-1} \\
& {[(z, \tau)] \mapsto\left([(z, \tau)], \sum_{k=1}^{g}\left(\sigma_{k}\left(a^{\prime}\right)+\sigma_{k}(a) \overline{\tau_{k}}\right) \bar{u}_{\tau, k}\right) .}
\end{aligned}
$$

\subsection{Champs de vecteurs}

La trivialisation $\Psi$ permet d'associer à tout élément $\theta$ de $\Pi_{\mathbb{C}}$ un champ de vecteurs $\mathcal{C}^{\infty}$ complexe sur $A^{\prime}$ que l'on note (abusivement) aussi $\theta$. Pour $\gamma=\left(a^{\prime}, a\right) \in \Pi$, les champs de vecteurs associés à $\gamma^{-1,0}$ et $\gamma^{0,-1}$ sont :

$$
\gamma^{-1,0}=\sum_{k=1}^{g}\left(\sigma_{k}\left(a^{\prime}\right)+\sigma_{k}(a) \tau_{k}\right) \partial_{z_{k}} \quad \text { et } \quad \gamma^{0,-1}=\sum_{k=1}^{g}\left(\sigma_{k}\left(a^{\prime}\right)+\sigma_{k}(a) \overline{\tau_{k}}\right) \partial_{\overline{z_{k}}} .
$$

\subsection{La forme de polarisation}

On déduit de la trivialisation $\Psi$ une décomposition du fibré tangent $\mathcal{C}^{\infty}$-complexe de $A^{\prime}$ : $T_{\mathbb{C}} A^{\prime}=\left(q^{\prime}\right)^{*} T_{\mathbb{C}} \mathfrak{H}^{g} \oplus\left(A^{\prime} \times \Pi_{\mathbb{C}}\right)$. La projection de $T A^{\prime}$ sur $\left(A^{\prime} \times \Pi_{\mathbb{C}}\right)$ induit une forme différentielle à valeurs dans $\left(A^{\prime} \times \Pi_{\mathbb{C}}\right)$ notée $\nu$.

\section{Notations $4.2-$}

- $D(d \tau)$ (resp. $D(d \bar{\tau})$ ) est la matrice diagonale de formes différentielles dont la diagonale est $\left(d \tau_{1}, \ldots, d \tau_{g}\right)$ (resp. $\left.\left(d \overline{\tau_{1}}, \ldots, d \overline{\tau_{g}}\right)\right)$.

- $u_{k}\left(\right.$ resp. $\left.\bar{u}_{k}\right)$ est la section de $\left(A^{\prime} \times \Pi_{\mathbb{C}}\right)$ au dessus de $A^{\prime} u_{k}:[(z, \tau)] \mapsto u_{\tau, k}$ (resp. $\bar{u}_{k}:[(z, \tau)] \mapsto$ $\left.\bar{u}_{\tau, k}\right)$ pour $k \in\{1, \ldots, g\}$.

Au prix d'un calcul faisant intervenir l'expression explicite de $\Psi^{-1}$ obtenue précédemment, on obtient l'expression en coordonnées de $\nu$ suivante :

$$
\nu=(d z-(z-\bar{z}) D(T(\tau)) D(d \tau))\left(\begin{array}{c}
u_{1} \\
\vdots \\
u_{g}
\end{array}\right)+(d \bar{z}-(z-\bar{z}) D(T(\tau)) D(d \bar{\tau}))\left(\begin{array}{c}
\bar{u}_{1} \\
\vdots \\
\bar{u}_{g}
\end{array}\right) .
$$

Soit $\tau \in \mathfrak{H}^{g}$. La matrice de Gram de l'accouplement $<\cdot, \cdot>_{\mathbb{C}}: \Pi_{\mathbb{C}} \wedge \Pi_{\mathbb{C}} \rightarrow \mathbb{C}$ (obtenu en étendant par linéarité $<\cdot, \cdot>)$ dans la base $\left(u_{\tau, 1}, \ldots, u_{\tau, g}, \bar{u}_{\tau, 1}, \ldots, \bar{u}_{\tau, g}\right)$ est 


$$
\left(<u_{\tau, k}, \bar{u}_{\tau, l}>_{\mathbb{C}}\right)_{1 \leq k, l \leq g}=-2 \pi i T(\tau)
$$

La forme de polarisation $\omega:=\frac{1}{2}<\nu, \nu>_{\mathbb{C}}$ est donc :

$$
\omega=-2 \pi i \sum_{k=1}^{g} \underbrace{\left(d z_{k}-t_{k}\left(z_{k}-\overline{z_{k}}\right) d \tau_{k}\right)}_{\eta_{k}^{1}} \wedge \underbrace{\left(t_{k}\left(d \overline{z_{k}}-t_{k}\left(z_{k}-\overline{z_{k}}\right) d \overline{\tau_{k}}\right)\right.}_{\eta_{k}^{2}} .
$$

\subsection{Normes}

Soient $\tau \in \mathfrak{H}^{g}$ et $\gamma=\left(a^{\prime}, a\right) \in \Pi$. De $\left(<u_{\tau, k}, \bar{u}_{\tau, l}>_{\mathbb{C}}\right)_{1 \leq k, l \leq g}=-2 \pi i T(\tau)$ et des expressions obtenues pour $\gamma^{-1,0}$ et $\gamma^{0,1}$ dans la partie 4.2, on déduit que l'image de $\tau$ par la fonction $\rho(\gamma)=$ $<\gamma^{-1,0}, \gamma^{0,1}>: \mathfrak{H}^{g} \rightarrow \mathbb{R}$ est

$$
\rho(\gamma)(\tau)=-2 \pi i \sum_{k=1}^{g} t_{k}\left|\sigma_{k}\left(a^{\prime}\right)+\sigma_{k}(a) \tau_{k}\right|^{2} .
$$

\subsection{Les fonctions $\chi_{\gamma}(\gamma \in \Pi)$}

Soit $\tau \in \mathfrak{H}^{g}$. L'isomorphisme de $\mathbb{R}$-vectoriels $\varphi_{\tau} \otimes \mathbb{R}: \Pi_{\mathbb{R}} \rightarrow \mathbb{C}^{g}$ induit par passage au quotient un isomorphisme $\mathcal{C}^{\infty}$-réel de tores réels $\overline{\varphi_{\tau} \otimes \mathbb{R}}: \Pi_{\mathbb{R}} / \Pi \rightarrow A_{\tau}^{\prime}$.

Soit $\gamma=\left(a^{\prime}, a\right) \in \Pi$. L'image de $[(z, \tau)] \in A^{\prime}$ par la fonction $\mathcal{C}^{\infty}$-réelle $\chi_{\gamma}: A^{\prime} \rightarrow \mathbb{C}$ est donnée par :

$$
\chi_{\gamma}([(z, \tau)])=\exp \left(<\gamma,{\overline{\varphi_{\tau} \otimes \mathbb{R}^{-1}}}^{\left.-z)>_{\mathbb{C}}\right)}\right.
$$

\subsection{Dérivées de Lie successives de la forme vol et effet du produit intérieur}

Soit $\alpha \in \mathbb{N}^{\geq 1}$ et soit $\gamma=\left(a^{\prime}, a\right) \in \Pi \backslash\{0\}$. On explicite tout d'abord $\frac{1}{\left(\rho(\gamma)-L_{\gamma^{-1,0}}\right)^{\alpha}} v o l$, où vol $:=(-1)^{g}(g !)^{-1} \omega^{g}$. On rappelle que $L_{\gamma^{-1,0}}$ désigne la dérivée de Lie du champ de vecteurs $\gamma^{-1,0}$ et que $L_{\gamma^{-1,0}}^{k} \omega^{g}=0$ si $k>2 g$ (cf. [L, Prop. 3.2.2]). À l'aide de l'expression de $\omega$ de la Partie 4.4, on obtient :

$$
\begin{aligned}
\frac{1}{\left(\rho(\gamma)-L_{\gamma^{-1,0}}\right)^{\alpha}} \operatorname{vol} & =\frac{(2 \pi i)^{g}}{\left(\rho(\gamma)-L_{\gamma^{-1,0}}\right)^{\alpha}} \eta_{1}^{1} \wedge \eta_{1}^{2} \wedge \eta_{2}^{1} \wedge \eta_{2}^{2} \wedge \cdots \wedge \eta_{g}^{1} \wedge \eta_{g}^{2} \\
& =\sum_{n=0}^{2 g} \frac{(2 \pi i)^{g} C_{n+\alpha-1}^{\alpha-1}}{\rho(\gamma)^{n+\alpha}}\left(L_{\gamma^{-1,0}}\right)^{n} \eta_{1}^{1} \wedge \eta_{1}^{2} \wedge \eta_{2}^{1} \wedge \eta_{2}^{2} \wedge \cdots \wedge \eta_{g}^{1} \wedge \eta_{g}^{2}
\end{aligned}
$$

Pour tout $n \in\{0, \ldots, 2 g\}$, on note $\mathcal{L}_{n}$ l'ensemble

$$
\left\{\left(L_{1}, \ldots, L_{2 g}\right) \in\left\{\mathrm{Id}, L_{\gamma^{-1,0}}\right\}^{2 g} \mid \sharp\left(\left\{k \in\{1, \ldots, 2 g\} \mid L_{k}=L_{\gamma^{-1,0}}\right\}\right)=n\right\} .
$$

Puisque $\left(L_{\gamma^{-1,0}}\right)^{2} \eta_{k}^{1}=\left(L_{\gamma^{-1,0}}\right)^{2} \eta_{k}^{2}=0$ pour tout $k \in\{1, \ldots, g\}$, , on a :

$$
\left(L_{\gamma^{-1,0}}\right)^{n} \eta_{1}^{1} \wedge \eta_{1}^{2} \wedge \cdots \wedge \eta_{g}^{1} \wedge \eta_{g}^{2}=\sum_{\left(L_{1}, \ldots, L_{2 d}\right) \in \mathcal{L}_{n}} L_{1} \eta_{1}^{1} \wedge L_{2} \eta_{1}^{2} \wedge \cdots \wedge L_{2 g-1} \eta_{g}^{1} \wedge L_{2 g} \eta_{g}^{2} .
$$


On vérifie alors que pour tout $k \in\{1, \ldots, g\}$ :

$$
L_{\gamma^{-1,0}} \eta_{k}^{1}=-t_{k}\left(\sigma_{k}\left(a^{\prime}\right)+\sigma_{k}(a) \overline{\tau_{k}}\right) d \tau_{k} \quad \text { et } \quad L_{\gamma^{-1,0}} \eta_{k}^{2}=-t_{k}^{2}\left(\sigma_{k}\left(a^{\prime}\right)+\sigma_{k}(a) d \overline{\tau_{k}}\right) .
$$

On a ainsi une expression en coordonnées de $\frac{1}{\left(\rho(\gamma)-L_{\gamma^{-1,0}}\right)^{\alpha}}$ vol. On calcule maintenant $i_{\gamma^{0,-1}} \frac{1}{\left(\rho(\gamma)-L_{\gamma^{-1,0}}\right)^{\alpha}} \operatorname{vol}\left(i_{\gamma^{0,-1}}\right.$ désigne l'opérateur de contraction par le champ de vecteurs $\left.\gamma^{0,-1}\right)$. Compte tenu de ce qui précède, il suffit d'expliciter $i_{\gamma^{0,-1}} L_{1} \eta_{1}^{1} \wedge L_{2} \eta_{1}^{2} \wedge \cdots \wedge L_{2 g-1} \eta_{g}^{1} \wedge L_{2 g} \eta_{g}^{2}$ pour tout $n \in\{0, \ldots, 2 g\}$ et $\left(L_{1}, . ., L_{2 g}\right) \in \mathcal{L}_{n}$.

Soient $n \in\{0, \ldots, 2 g\}$ et $\left(L_{1}, . ., L_{2 g}\right) \in \mathcal{L}_{n}$.

$$
\begin{aligned}
i_{\gamma^{0,-1}} L_{1} \eta_{1}^{1} \wedge L_{2} \eta_{1}^{2} \wedge \cdots \wedge L_{2 g-1} \eta_{g}^{1} \wedge L_{2 g} \eta_{g}^{2} & =\left(i_{\gamma^{0,-1}} L_{1} \eta_{1}^{1}\right) L_{2} \eta_{1}^{2} \wedge \cdots \wedge L_{2 g-1} \eta_{g}^{1} \wedge L_{2 g} \eta_{g}^{2} \\
& -\left(i_{\gamma^{0,-1}} L_{2} \eta_{1}^{2}\right) L_{1} \eta_{1}^{1} \wedge L_{3} \eta_{2}^{1} \wedge \cdots \wedge L_{2 g-1} \eta_{g}^{1} \wedge L_{2 g} \eta_{g}^{2} \\
& +\cdots \\
& -\left(i_{\gamma^{0,-1}} L_{2 g} \eta_{g}^{2}\right) L_{1} \eta_{1}^{1} \wedge L_{2} \eta_{1}^{2} \wedge \cdots \wedge L_{2 g-1} \eta_{g}^{1} .
\end{aligned}
$$

De plus, pour tout $k \in\{1, \ldots, g\}$ :

$$
i_{\gamma^{0-1}} \eta_{k}^{2}=t_{k}\left(\sigma_{k}\left(a^{\prime}\right)+\sigma_{k}(a) \overline{\tau_{k}}\right) \quad \text { et } \quad i_{\gamma^{0-1}} \eta_{k}^{1}=i_{\gamma^{0-1}} L_{\gamma^{-1,0}} \eta_{k}^{1}=i_{\gamma^{0-1}} L_{\gamma^{-1,0}} \eta_{k}^{2}=0 .
$$

\subsection{Détermination des classes d'Eisenstein au niveau topologique}

On fixe $\left(b^{\prime}, b\right) \in\left(N^{-1} \mathfrak{a}^{\vee} \oplus N^{-1} \mathfrak{a}\right) \backslash\{0\}$ et $l \in \mathbb{N}^{>2 g}$.

De la définition du morphisme $\mathcal{P}_{\omega}$ de Levin $[\mathrm{L}$, Thm 3.4.4] et de la Partie 5 de [B2] et d'un calcul élémentaire, on déduit que $\operatorname{For}\left(\mathcal{E}_{x_{b^{\prime}, b}}^{l}\right) \in H_{\mathrm{Betti}}^{2 g-1}\left(S^{0}(\mathbb{C}), \overline{\left(\operatorname{Sym}^{l} \mathcal{H}\right)(g)}\right)$ coïncide avec la classe de cohomologie induite par la forme différentielle

$$
(-1)^{l}(l+2 g) \sum_{\gamma \in \Pi \backslash\{0\}} x_{b^{\prime}, b}^{*} g_{l+1, \gamma}^{\prime} \in \Gamma\left(\mathfrak{H}^{g}, \Omega_{\mathfrak{H}^{g}}^{2 g-1} \otimes \operatorname{Sym}^{l} \Pi_{\mathbb{C}}\right)
$$

où $g_{l+1, \gamma}^{\prime}=\chi_{\gamma} i_{\gamma^{0,-1}} \frac{1}{\left(\rho(\gamma)-L_{\gamma^{-1,0}}\right)^{l+1}} \operatorname{vol} \otimes\left(\gamma^{0,-1}\right)^{l}$, pour $\gamma \in \Pi \backslash\{0\}$.

Soit $\gamma=\left(a^{\prime}, a\right) \in \Pi$ et $k \in\{1, \ldots, g\}$. On explicite maintenant $x_{b^{\prime}, b}^{*} g_{l+1, \gamma}^{\prime}$ à l'aide des calculs effectués précédemment.

On a les relations suivantes

$$
\begin{array}{ll}
x_{b^{\prime}, b}^{*} \eta_{k}^{1}=x_{b^{\prime}, b}^{*} \eta_{k}^{2}=0 & x_{b^{\prime}, b}^{*} L_{\gamma^{-1,0}} \eta_{k}^{1}=-t_{k}\left(\sigma_{k}\left(a^{\prime}\right)+\sigma_{k}(a) \overline{\tau_{k}}\right) \\
x_{b^{\prime}, b}^{*} L_{\gamma^{-1,0}} \eta_{k}^{2}=-t_{k}^{2}\left(\sigma_{k}\left(a^{\prime}\right)+\sigma_{k}(a) \tau_{k}\right) d \overline{\tau_{k}} & x_{b^{\prime}, b}^{*} i_{\gamma^{0-1}} \eta_{k}^{2}=t_{k}\left(\sigma_{k}\left(a^{\prime}\right)+\sigma_{k}(a) \overline{\tau_{k}}\right) .
\end{array}
$$

et par suite

$$
(-1)^{l}(l+2 g) x_{b^{\prime}, b}^{*} i_{\gamma^{0,-1}} \frac{1}{\left(\rho(\gamma)-L_{\gamma^{-1,0}}\right)^{l+1}} v o l=\frac{(-1)^{l+1}(2 g+l) !(2 \pi i)^{g}}{l ! \rho(\gamma)^{2 g+l}} \sum_{k=1}^{g} \nu_{k}
$$

avec

$\nu_{k}:=t_{k}^{2}\left(\sigma_{k}\left(a^{\prime}\right)+\sigma_{k}(a) \overline{\tau_{k}}\right)^{2}\left(\prod_{j \neq k} t_{j}^{3}\left|\sigma_{j}\left(a^{\prime}\right)+\sigma_{j}(a) \tau_{j}\right|^{2}\right) d \tau_{1} \wedge d \overline{\tau_{1}} \wedge \cdots \wedge d \tau_{k} \wedge \widehat{d \overline{\tau_{k}}} \wedge \cdots \wedge d \tau_{g} \wedge d \overline{\tau_{g}}$, 
pour $k \in\{1, \ldots, g\}$. En effet, seule reste la contribution de $i_{\gamma^{0,-1}} L_{\gamma^{-1,0}}^{2 g-1}$ vol. On a également

$$
x_{b^{\prime}, b}^{*} \chi_{\gamma}=\exp \left(<\gamma,\left(b^{\prime}, b\right)>\right)=\exp \left(2 \pi i \operatorname{Tr}_{L}\left(a^{\prime} b-a b^{\prime}\right)\right) .
$$

De plus, on a explicité $\gamma^{-1,0}$ relativement à la base $\left(\bar{u}_{\tau, 1}, \ldots, \bar{u}_{\tau, g}\right)$, mais aussi, la base $\left(\bar{u}_{\tau, 1}, \ldots, \bar{u}_{\tau, g}\right)$ relativement à une $\mathbb{Z}$-base de $\mathfrak{a}^{\vee} \oplus \mathfrak{a}$ (cf. Partie 4.2). On en déduit l'expression de $\gamma^{-1,0}$ dans $\mathbb{C}^{g} \oplus \mathbb{C}^{g}$, via l'isomorphisme $\iota:\left(\mathfrak{a}^{\vee} \oplus \mathfrak{a}\right) \otimes_{\mathbb{Z}} \mathbb{C} \stackrel{\sim}{\rightarrow} \mathbb{C}^{g} \oplus \mathbb{C}^{g}$ induit par les plongements $\left(\sigma_{k}\right)_{1 \leq k \leq g}$ :

$$
\gamma^{-1,0}=\left(\left(-\left(t_{k} \overline{\tau_{k}}\left(\sigma_{k}\left(a^{\prime}\right)+\sigma_{k}^{\prime}(a) \tau_{k}\right)\right)_{1 \leq k \leq g},\left(t_{k}\left(\sigma_{k}\left(a^{\prime}\right)+\sigma_{k}^{\prime}(a) \tau_{k}\right)_{1 \leq k \leq g}\right) .\right.\right.
$$

On rassemble les résultats obtenus dans la Proposition suivante.

Proposition 4.3 - La $(g, g-1)$-forme différentielle sur $\mathfrak{H}^{g}$ à valeurs dans $S y m^{l} \Pi_{\mathbb{C}}$

$$
(2 \pi i)^{g} \frac{(-1)^{l+1}(2 g+l) !}{l !} \sum_{\left(a^{\prime}, a\right) \in \mathfrak{a}^{\vee} \oplus \mathfrak{a} \backslash\{0\}} \sum_{k=1}^{g} \frac{\exp \left(2 \pi i \operatorname{Tr}_{L}\left(a^{\prime} b-b^{\prime} a\right)\right)}{\rho\left(a^{\prime}, a\right)^{2 g+l}} f_{k}\left(a^{\prime}, a, \tau\right) \mu_{k} \otimes h\left(a^{\prime}, a, \tau\right)^{l}
$$

où pour tout $\left(a^{\prime}, a\right) \in \mathfrak{a}^{\vee} \oplus \mathfrak{a}, \tau \in \mathfrak{H}^{g}, k \in\{1, \ldots, g\}$, on note $t_{k}:=\left(\tau_{k}-\overline{\tau_{k}}\right)^{-1}$ et

$$
\begin{aligned}
& \rho\left(a^{\prime}, a\right):=-2 \pi i \sum_{j=1}^{g} t_{j}\left|\sigma_{j}\left(a^{\prime}\right)+\sigma_{j}(a) \tau_{j}\right|^{2}, \\
& f_{k}\left(a^{\prime}, a, \tau\right):=t_{k}^{2}\left(\sigma_{k}\left(a^{\prime}\right)+\sigma_{k}(a) \overline{\tau_{k}}\right)^{2} \prod_{j \neq k} t_{j}^{3}\left|\sigma_{j}\left(a^{\prime}\right)+\sigma_{j}(a) \tau_{j}\right|^{2}, \\
& \mu_{k}:=d \tau_{1} \wedge d \overline{\tau_{1}} \wedge \cdots \wedge d \tau_{k} \wedge \widehat{d \overline{\tau_{k}}} \wedge \cdots \wedge d \tau_{g} \wedge d \overline{\tau_{g}} \in \Gamma\left(\mathfrak{H}^{g}, \Omega_{\mathfrak{H}^{g}, \mathbb{C}}^{2 g-1}\right),
\end{aligned}
$$

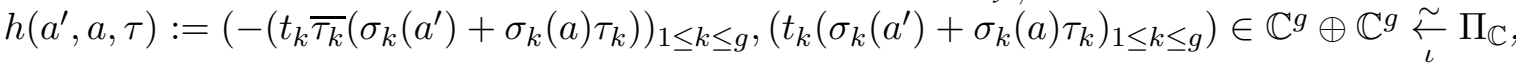

induit une classe de cohomologie dans $H_{\text {Betti }}^{2 g-1}\left(\Lambda(\mathfrak{a}, N) \backslash \mathfrak{H}^{g},\left(\overline{\left(\operatorname{Sym}^{l} \mathcal{H}\right)(d)}\right)_{\mathbb{C}}\right)$ qui coïncide avec For $\left(\mathcal{E} i s_{x_{b^{\prime}, b}}^{l}\right)$.

\section{Détermination du résidu en l' $\infty$ des classes d'Eisenstein}

On fixe $b^{\prime} \in N^{-1} \mathfrak{a}^{-1}, b \in\left(N^{-1} \mathfrak{a}\right) \backslash \mathfrak{a}, \lambda \in \mathbb{N} \geq 3$.

On note $\operatorname{pr}_{\text {res }}: \operatorname{Sym}^{\lambda g}\left(\mathbb{C}^{g} \oplus \mathbb{C}^{g}\right) \rightarrow \mathbb{C}$ la projection qui correspond au niveau complexe à la projection de

$$
\operatorname{Sym}^{\lambda g} V(\overline{\mathbb{Q}}) \rightarrow \operatorname{Sym}^{\lambda g} V(\overline{\mathbb{Q}}) / W
$$

introduite précédemment (cf. Remarque 3.3).

Le morphisme $\overline{\operatorname{Res}_{\infty}^{\lambda g}} \otimes \mathbb{C}$, obtenu à partir de $\overline{\operatorname{Res}_{\infty}^{\lambda g}}$ par extension des scalaires de $\mathbb{Q}$ à $\mathbb{C}$, est donné par :

$$
\begin{aligned}
\overline{\left.\operatorname{Res}_{\infty}^{\lambda g} \otimes \mathbb{C}: \quad H_{\operatorname{Betti}}^{2 g-1}\left(\Lambda(\mathfrak{a}, N) \backslash \mathfrak{H}^{g}, \overline{\left(\operatorname{Sym}^{\lambda g} \mathcal{H}(g)\right.}\right)_{\mathbb{C}}\right)} & \rightarrow \mathbb{C} \\
{\left[\theta \otimes\left[v_{1} \otimes . . \otimes v_{\lambda g}\right]\right] } & \mapsto\left(\frac{1}{(2 \pi i)^{g}} \int_{\Lambda(\mathfrak{a}, N, \infty) \backslash D_{r}} \theta\right) \times p r_{r e s}\left(\left[v_{1} \otimes . . \otimes v_{\lambda g}\right]\right) .
\end{aligned}
$$

où $D_{r}:=\left\{\left(\tau_{1}, \ldots, \tau_{g}\right) \in \mathfrak{H}^{g} \mid \prod_{k=1}^{g} \Im\left(\tau_{k}\right)=r\right\}$ pour un nombre réel $r \gg 0$. Ce morphisme respecte les structures rationnelles sous-jacentes. Cette partie est consacrée au calcul de

$$
\begin{aligned}
\operatorname{Res}_{\infty}^{\lambda g}\left(\mathcal{E} i s_{x_{b^{\prime}, b}}^{\lambda g}\right) & =\overline{\operatorname{Res}_{\infty}^{\lambda g}}\left(F \operatorname{For}\left(\mathcal{E} i s_{x_{b^{\prime}, b}}^{\lambda g}\right)\right. \\
& \left.=\overline{\operatorname{Res}_{\infty}^{\lambda g}} \otimes \mathbb{C}\left(F \operatorname{Er} i s_{x_{b^{\prime}, b}}^{\lambda g}\right)\right) \\
& =(-1)^{\lambda g}(\lambda+2) g \sum_{\gamma \in \mathfrak{a}^{\vee} \oplus \mathfrak{a} \backslash\{0\}} \overline{\operatorname{Res}_{\infty}^{\lambda g}} \otimes \mathbb{C}\left(x_{b^{\prime}, b}^{*} g_{\lambda g+1, \gamma}^{\prime}\right) .
\end{aligned}
$$


D'après la Proposition 4.3 , le nombre rationnel $\operatorname{Res}_{\infty}^{\lambda g}\left(\mathcal{E} i s_{x_{b^{\prime}, b}}^{\lambda g}\right)$ est égal à :

$$
\frac{(-1)^{\lambda g+1}((\lambda+2) g) !}{(\lambda g) !} \sum_{k=1}^{g} \sum_{\left(a^{\prime}, a\right) \in \mathfrak{a}^{\vee} \oplus \mathfrak{a} \backslash\{0\}} \exp \left(2 \pi i \operatorname{Tr}_{L}\left(a^{\prime} b-b^{\prime} a\right)\right) I_{a^{\prime}, a, k}
$$

où

$$
I_{a^{\prime}, a, k}:=\int_{\Lambda(\mathfrak{a}, N, \infty) \backslash D_{r}} \frac{\prod_{j=1}^{g} t_{j}^{\lambda}\left(\sigma_{j}\left(a^{\prime}\right)+\sigma_{j}(a) \tau_{j}\right)^{\lambda}}{\left(\sum_{j=0}^{g} 2 \pi i t_{j}\left|\sigma_{j}\left(a^{\prime}\right)+\sigma_{j}(a) \tau_{j}\right|^{2}\right)^{(\lambda+2) g}} \nu_{k}
$$

avec

$\nu_{k}=t_{k}^{2}\left(\sigma_{k}\left(a^{\prime}\right)+\sigma_{k}(a) \overline{\tau_{k}}\right)^{2}\left(\prod_{j \neq k} t_{j}^{3}\left|\sigma_{j}\left(a^{\prime}\right)+\sigma_{j}(a) \tau_{j}\right|^{2}\right) d \tau_{1} \wedge d \overline{\tau_{1}} \wedge \cdots \wedge d \tau_{k} \wedge \widehat{d \overline{\tau_{k}}} \wedge \cdots \wedge d \tau_{g} \wedge d \overline{\tau_{g}}$, pour $k \in\{1, \ldots, g\}$.

- Étape 1 : Calcul de $I_{1, k}:=\sum_{a \in \mathfrak{a} \backslash\{0\}} \sum_{a^{\prime} \in \mathfrak{a}^{\vee}} \exp \left(2 \pi i \operatorname{Tr}_{L}\left(a^{\prime} b-b^{\prime} a\right)\right) I_{a^{\prime}, a, k}$, pour $k \in\{1, \ldots, g\}$.

i) Dans $I_{a^{\prime}, a, k}$, on peut effectuer $(a \neq 0)$ le changement de variables

$$
\tau_{j}^{\prime}=\tau_{j}+\frac{\sigma_{j}\left(a^{\prime}\right)}{\sigma_{j}(a)}, \text { pour } 1 \leq j \leq g
$$

pour observer que $I_{a^{\prime}, a, k}=I_{0, a, k}$.

ii) Comme $b \notin \mathfrak{a}$, il existe $a^{\prime} \in \mathfrak{a}^{\vee}$ tel que :

$$
\operatorname{Tr}_{L}\left(a^{\prime} b\right) \notin \mathbb{Z}
$$

iii) De i) et ii), on déduit que $I_{1, k}=0$.

- Étape 2: Simplification de $I_{2, k}:=\sum_{a^{\prime} \in \mathfrak{a}^{\vee} \backslash\{0\}} \exp \left(2 \pi i \operatorname{Tr}_{L}\left(a^{\prime} b\right)\right) I_{a^{\prime}, 0, k}$, pour $k \in\{1, \ldots, g\}$.

On ne calcule que $I_{2, k}$ que pour $k=1$, la méthode étant analogue pour les autres valeurs de $k$ et on peut supposer $r=1$. De nombreuses simplifications apparaissent lorsque $a=0$ dans $I_{a^{\prime}, a, k}$. On note $x_{k}:=\Re\left(\tau_{k}\right)$ et $y_{k}=\Im\left(\tau_{k}\right)$, pour $k \in\{1, \ldots, g\}$. On a :

$$
I_{2, k}=\sum_{a^{\prime} \in \mathfrak{a}^{\vee} \backslash\{0\}} \exp \left(2 \pi i \operatorname{Tr}_{L}\left(a^{\prime} b\right)\right) \frac{(-1)^{g-1} \mathrm{~N}_{L}\left(a^{\prime}\right)^{\lambda+2}}{(2 \pi i)^{(l+2 g)}} J_{a^{\prime}}
$$

où

$$
J_{a^{\prime}}=\int_{\Lambda(\mathfrak{a}, N, \infty) \backslash D_{1}} \frac{1}{\left(\sum_{j=0}^{g} \frac{\sigma_{j}\left(a^{\prime}\right)^{2}}{y_{j}}\right)^{(\lambda+2) g}}\left(d x_{1}+i d y_{1}\right) \wedge d x_{2} \wedge \frac{d y_{2}}{y_{2}} \wedge \cdots \wedge d x_{g} \wedge \frac{d y_{g}}{y_{g}}
$$


De la relation $y_{1} \ldots y_{g}=1$ vérifiée par $\left(\tau_{1}, \ldots, \tau_{g}\right) \in D_{1}$, on déduit :

$$
\begin{aligned}
J_{a^{\prime}} & =\int_{\Lambda(\mathfrak{a}, N, \infty) \backslash D_{1}} \frac{1}{\left(\sum_{j=0}^{g} \frac{\sigma_{j}\left(a^{\prime}\right)^{2}}{y_{j}}\right)^{(\lambda+2) g}} d x_{1} \wedge d x_{2} \wedge \frac{d y_{2}}{y_{2}} \wedge \cdots \wedge d x_{g} \wedge \frac{d y_{g}}{y_{g}} \\
& =\sum_{a^{\prime} \in\left(\mathfrak{a}^{\vee} \backslash\{0\}\right) / U_{L, N}} \frac{(-1)^{g-1} \exp \left(2 \pi i \operatorname{Tr}_{L}\left(a^{\prime} b\right)\right) \mathrm{N}_{L}\left(a^{\prime}\right)^{\lambda+2}}{(2 \pi i)^{(\lambda+2) g}} \\
& \times \sum_{\varepsilon \in U_{L, N}} \int_{\Lambda(\mathfrak{a}, N, \infty) \backslash D_{1}} \frac{1}{\left(\sum_{j=0}^{g} \frac{\sigma_{j}\left(\varepsilon a^{\prime}\right)^{2}}{y_{j}}\right)^{(\lambda+2) g}} d x_{1} \wedge d x_{2} \wedge \frac{d y_{2}}{y_{2}} \wedge \cdots \wedge d x_{g} \wedge \frac{d y_{g}}{y_{g}} \\
& =\sum_{a^{\prime} \in\left(\mathfrak{a}^{\vee} \backslash\{0\}\right) / U_{L, N}} \frac{(-1)^{g-1} \exp \left(2 \pi i \operatorname{Tr}_{L}\left(c b^{\prime}\right)\right) \mathrm{N}_{L}\left(a^{\prime}\right)^{\lambda+2}}{(2 \pi i)^{(\lambda+2) g}} \operatorname{vol}\left(N \mathfrak{d}_{L}^{-1} \mathfrak{a}^{-2}\right) K_{a^{\prime}}
\end{aligned}
$$

où

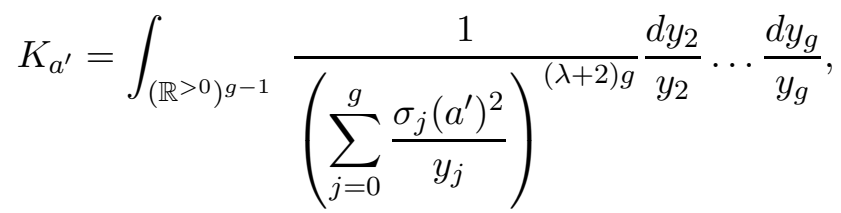

pour $a^{\prime} \in\left(\mathfrak{a}^{\vee} \backslash\{0\}\right) / U_{L, N}$. Pour établir la dernière égalité, on utilise le Lemme $2.10_{1}$ de $[\mathrm{F}]$ et l'injectivité de l'homomorphisme de groupes $U_{L, N} \rightarrow U_{L, N}, \varepsilon \mapsto \varepsilon^{2}$.

- Étape 3: Calcul de $K_{a^{\prime}}$, pour $a^{\prime} \in\left(\mathfrak{a}^{\vee} \backslash\{0\}\right) / U_{L, N}$.

On calcule en fait $\Gamma((\lambda+2) g) K_{a^{\prime}}$ :

$\Gamma((\lambda+2) g) K_{a^{\prime}}=\int_{(\mathbb{R}>0)^{g}} u^{(\lambda+2) g} \exp \left(-u\left(y_{2} \ldots y_{g} \sigma_{1}\left(a^{\prime}\right)^{2}+\frac{\sigma_{2}\left(a^{\prime}\right)^{2}}{y_{2}}+\cdots+\frac{\sigma_{g}\left(a^{\prime}\right)^{2}}{y_{g}}\right)\right) \frac{d u}{u} \frac{d y_{2}}{y_{2}} \ldots \frac{d y_{g}}{y_{g}}$.

On effectue ensuite le changement de variables :

$$
\begin{gathered}
u_{1}=u y_{2} \ldots y_{g} \\
u_{2}=u / y_{2} \\
\vdots \\
u_{g}=u / y_{g}
\end{gathered}
$$

et on trouve :

$$
\begin{aligned}
\Gamma((\lambda+2) g) K_{a^{\prime}} & =\left(\int_{\mathbb{R}>0} u_{1}^{\lambda+2} \exp \left(-\sigma_{1}\left(a^{\prime}\right)^{2} u_{1}\right) \frac{d u_{1}}{u_{1}}\right) \ldots\left(\int_{\mathbb{R}^{>0}} u_{g}^{\lambda+2} \exp \left(-\sigma_{g}\left(a^{\prime}\right)^{2} u_{g}\right) \frac{d u_{g}}{u_{g}}\right) \\
& =\frac{\Gamma(\lambda+2)^{g}}{\mathrm{~N}_{L}\left(a^{\prime}\right)^{2(\lambda+2)}} .
\end{aligned}
$$

Ainsi $K_{a^{\prime}}=\frac{((\lambda+1) !)^{g}}{((\lambda+2) g-1) ! \mathrm{N}_{L}\left(a^{\prime}\right)^{2(\lambda+2)}}$. 
Notation 5.1 - Pour $\beta \in\left(N^{-1} \mathfrak{a}\right) \backslash \mathfrak{a}, s \in \mathbb{C}$ tel que $\Re(s)>1$, on définit $\mathfrak{L}(\mathfrak{a}, N, \beta, s)$ par

$$
\mathfrak{L}(\mathfrak{a}, N, \beta, s):=\sum_{a^{\prime} \in\left(\mathfrak{a}^{\vee} \backslash\{0\}\right) / U_{L, N}} \frac{\exp \left(2 \pi i \operatorname{Tr}_{L}\left(a^{\prime} \beta\right)\right)}{N_{L}\left(a^{\prime}\right)^{s}} .
$$

Du calcul que l'on vient d'achever, on déduit le Théorème suivant.

Théorème 5.2 - Soient $\lambda \in \mathbb{N} \geq 3, b^{\prime} \in N^{-1} \mathfrak{a}^{\vee}$ et $b \in\left(N^{-1} \mathfrak{a}\right) \backslash \mathfrak{a}$.

$$
\operatorname{Res}_{\infty}^{\lambda g}\left(\mathcal{E} i s_{x_{b^{\prime}, b}}^{\lambda g}\right)=\frac{(-1)^{(\lambda+1) g}((\lambda+1) !)^{g}(\lambda+2) g^{2} N^{g}}{(\lambda g) ! \mathcal{N}_{L}(\mathfrak{a})^{2}} \frac{1}{\sqrt{d_{L}}(2 \pi i)^{(\lambda+2) g}} \mathfrak{L}(\mathfrak{a}, N, b, \lambda+2)
$$

Ayant pris soin de respecter les structures rationnelles tout au long du calcul, on déduit de ce résultat le Théorème de Klingen-Siegel.

Corollaire 5.3 - Pour tout $\lambda \in \mathbb{N} \geq 6$ pair et $b \in\left(N^{-1} \mathfrak{a}\right) \backslash \mathfrak{a}$ :

$$
\mathfrak{L}(\mathfrak{a}, N, b, \lambda)(2 \pi i)^{-\lambda g} \sqrt{d_{L}} \in \mathbb{Q} \text {. }
$$

Corollaire 5.4 - Soient $\lambda \in \mathbb{N} \geq 6$ pair, $b^{\prime} \in N^{-1} \mathfrak{a}^{\vee}$ et $b \in\left(N^{-1} \mathfrak{a}\right) \backslash \mathfrak{a}$ tel que les idéaux entiers $N \mathcal{O}_{L}$ et $N b \mathfrak{a}^{-1}$ sont copremiers. Si $g \geq 2$, alors on $a$ :

$$
\mathcal{E} i s_{x_{b^{\prime}, b}}^{\lambda g} \neq 0 \text {. }
$$

Démonstration - On prouve que $\operatorname{Res}_{\infty}^{\lambda g}\left(\mathcal{E}_{i x_{b^{\prime}, b}}^{\lambda g}\right) \neq 0$ à l'aide du Théorème 5.2. On pose $\mathfrak{f}:=N \mathcal{O}_{L}$ et $\mathfrak{b}:=N b \mathfrak{a}^{-1}$. On introduit :

- l'ensemble $\mathcal{E}(\mathfrak{b}, \mathfrak{f})$ des idéaux entiers $\mathfrak{g}$ premiers à $\mathfrak{f}$ pour lesquels il existe $\mu \in L$ totalement positif et congru à 1 modulo $\mathfrak{f b}^{-1}$ tel que :

$$
\mathfrak{g}^{-1}=(\mu)
$$

- la fonction holomorphe $\zeta(\mathfrak{b}, \mathfrak{f}, \cdot)$ définie, pour $s \in \mathbb{C}$ tel que $\Re(s)>1$, par :

$$
\zeta(\mathfrak{b}, \mathfrak{f}, s):=\sum_{\mathfrak{g} \in \mathcal{E}(\mathfrak{b}, \mathfrak{f})} N_{L}(\mathfrak{g})^{-s},
$$

- $U_{L, N}^{+}$le sous-groupe de $U_{L, N}$ formé par les éléments de $U_{L, N}$ totalement positifs,

- la fonction holomorphe $\mathfrak{L}^{+}(\mathfrak{a}, N, b, \cdot)$ définie, pour $s \in \mathbb{C}$ tel que $\Re(s)>1$, par :

$$
\mathfrak{L}^{+}(\mathfrak{a}, N, b, s):=\sum_{a^{\prime} \in\left(\mathfrak{a}^{\vee} \backslash\{0\}\right) / U_{L, N}^{+}} \frac{\exp \left(2 \pi i \operatorname{Tr}_{L}\left(a^{\prime} b\right)\right)}{\left|\mathrm{N}_{L}\left(a^{\prime}\right)\right|^{s}} .
$$

La fonction $\zeta(\mathfrak{b}, \mathfrak{f}, \cdot)$ a un prolongement holomorphe sur $\mathbb{C}-\{1\}$. D'après une équation fonctionnelle pour $\mathfrak{L}^{+}\left(\mathcal{D}_{L}^{-1}, N, b, \cdot\right)$ établie par Siegel (cf. [Si, Formule (10)]), on a :

$$
\left[U_{L, N}: U_{L, N}^{+}\right] \mathfrak{L}\left(\mathcal{D}_{L}^{-1}, N, b^{\prime}, \lambda+2\right)=\mathfrak{L}^{+}\left(\mathcal{D}_{L}^{-1}, N, b^{\prime}, \lambda+2\right) \underset{\mathbb{R}^{x}}{\sim} \zeta(\mathfrak{b}, \mathfrak{f},-\lambda-1),
$$

et, comme $\lambda$ est pair, $\zeta(\mathfrak{b}, \mathfrak{f},-\lambda-1)$ est non nul (cf. [Ne, Theorem VII-5.9]). 


\section{Références}

[B1] D. Blottière, Dégénérescence des classes d'Eisenstein des familles modulaires de HilbertBlumenthal, Comptes Rendus Mathématique, Académie des Sciences, Paris, Volume 345, Issue 1, p. 5-10 (2007).

[B2] D. Blottière, Réalisation de Hodge du polylogarithme d'un schéma abélien, Prépublication disponible sur le serveur ArXiv (math.AG.0705.0880), 30 p..

[BW] J.I. Burgos, J. Wildeshaus, Hodge modules on Shimura varieties and their higher images in the Baily-Borel compactification, Ann. Sci. École Norm. Sup. 37, p. 363-413 (2004).

[F] E. Freitag, Hilbert Modular Forms, Springer-Verlag (1990).

[G] E.Z. Goren, Lectures on Hilbert modular varieties and modular forms, CRM Monograph Series, 14. American Mathematical Society, Providence, RI (2002).

[Ka] M. Kashiwara, A study of variations of mixed Hodge structure, Publ. RIMS, Kyoto Univ. 22, p. 991-1024 (1986).

[Ki1] G. Kings, $K$-theory elements for the polylogarithm of abelian schemes, J. reine angew. Math. 517, p. 103-116 (1999).

[Ki2] G. Kings, Degeneration of polylogarithms and special values of L-functions for totally real fields, Prépubilcation disponible sur le serveur ArXiv (math.NT.0510147), 33 p..

[Kl] H. Klingen, Über die Werte der Dedekindschen Zetafunktion, Math. Ann. 145, p. 265-272 (1962).

[Kn] A.W. Knapp Lie groups, Lie algebra, and Cohomology, Mathematical notes 34, Princeton Univ. Press (1988).

[L] A. Levin, Polylogarithmic Currents on Abelian Varieties, in A. Reznikov, N. Schappacher (eds), "Regulators in Analysis, Geometry and Number Theory", Proc. of the German-Israeli workshop held March 11-20 1996 at Landau Center, Jerusalem, Progress in Math. 171, Birkhäuser, p. 207-229 (2000).

[Ne] J. Neukirch, Algebraic Number Theory, Grundlehren der mathematischen Wissenschaften 322, Springer Verlag (1999).

[No] M. Nori, Some Eisenstein classes for the integral unimodular group, Proc. of the IMC Zürich, p. 690-696 (1994).

[P] R. Pink, Arithmetical compatification of mixed Shimura varieties, PhD Thesis, Bonn (1989).

[Sa] M. Saito, Mixed Hodge Modules, Publ. RIMS Kyoto Univ. 26, p. 221-233 (2000).

[Sc] R. Sczech, Eisenstein group cocycles for $G L_{n}$ and values of $L$-functions, Inv. Math. 113, p. 581-616 (1993).

[Si] C.L. Siegel, Über die Fourierschen Koeffizienten von Modulformen, Nach. Akad. Wiss. n ${ }^{\circ}$, p. $15-56$ (1970).

[W] J. Wildeshaus, Realizations of polylogarithms, L.N.M. 1650, Springer-Verlag Berlin (1997). 